\title{
High-Energy Ion Generation by Short Laser Pulses ${ }^{1}$
}

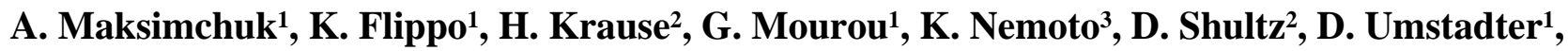 \\ R. Vane ${ }^{2}$, V. Yu. Bychenkov ${ }^{4}$, G. I. Dudnikova ${ }^{5}$, V. F. Kovalev 6 , K. Mima ${ }^{7}$, \\ V. N. Novikov ${ }^{4}$, Y. Sentoku ${ }^{8}$, and S. V. Tolokonnikov ${ }^{9}$ \\ ${ }^{1}$ Center for Ultrafast Optical Science, University of Michigan, Ann Arbor, MI 48109-2099, USA \\ ${ }^{2}$ Oak Ridge National Laboratory, Oak Ridge, TN 37831-6372, USA \\ ${ }^{3}$ Central Research Institute of Electric Power Industry, Tokyo 201-8511, Japan \\ ${ }^{4}$ Lebedev Physics Institute, Russian Academy of Sciences, Leninskii pr. 53, Moscow, 119991 Russia \\ ${ }^{5}$ Institute of Computational Technologies, Siberian Branch of Russian Academy of Sciences, Novosibirsk, 630090 Russia \\ ${ }^{6}$ Institute for Mathematical Modeling, Russian Academy of Sciences, Miusskaya pl. 4a, Moscow, 125047 Russia \\ ${ }^{7}$ Institute for Laser Engineering, Osaka University, 2-6 Yamada-oka, Suita, Osaka 565-0871, Japan \\ ${ }^{8}$ General Atomics, P.O. Box 85608, San Diego, California 92186, USA \\ ${ }^{9}$ Russian Research Centre Kurchatov Institute, pl. Kurchatova 1, Moscow, 123182 Russia \\ Received June 7, 2003; in final form, September 4, 2003
}

\begin{abstract}
This paper reviews the many recent advances at the Center for Ultrafast Optical Science (CUOS) at the University of Michigan in multi-MeV ion beam generation from the interaction of short laser pulses focused onto thin foil targets at intensities ranging from $10^{17}$ to $10^{19} \mathrm{~W} / \mathrm{cm}^{2}$. Ion beam characteristics were studied by changing the laser intensity, laser wavelength, target material, and by depositing a well-absorbed coating. We manipulated the proton beam divergence using shaped targets and observed nuclear transformation induced by high-energy protons and deuterons. Qualitative theoretical approaches and fully relativistic two-dimensional particle-in-cell simulations modeled energetic ion generation. Comparison with experiments sheds light on ion energy spectra for multi-species plasma, the dependences of ion-energy on preplasma scale length and solid density plasma thickness, and laser-triggered isotope yield. Theoretical predictions are also made with the aim of studying ion generation for high-power lasers with the energies expected in the near future, and for the relativistic intensity table-top laser, a prototype of which is already in operation at CUOS in the limits of severalcycle pulse duration and a single-wavelength spot size. (C) 2004 MAIK “Nauka/Interperiodica”.
\end{abstract}

\section{INTRODUCTION}

Developments in laser technology have enabled high power lasers to produce multi-terrawatt femtosecond pulses that allow the examination of the fundamental physics of ion acceleration at multi-MeV energies. At focal intensities of $I>10^{18} \mathrm{~W} / \mathrm{cm}^{2}$, high-intensity laser-triggered ion acceleration results in the formation of a multi-MeV beam propagating in the forward direction [1-4]. Experiments have already proven the possibility of transforming laser energy into collimated ultra-fast ion bunches with high efficiency (of up to several percent) when focusing ultra-short laser pulses of intensity $10^{19}-10^{20} \mathrm{~W} / \mathrm{cm}^{2}$ onto solid targets $[3,4]$. The mechanisms and characteristics of ions triggered by the interaction of a short laser pulse with plasma are of current interest because of their possible applications to novel neutron source development and isotope production. The proof-of-principle experiments with high energy lasers which should appear the near future intend to use ultra-intense laser pulses for ion beam generation with energies useful in proton therapy, fast ignition inertial confinement fusion, radiography, neu-

\footnotetext{
${ }^{1}$ This article was submitted by the authors in English.
}

tron sources, and the study of nuclear physics phenomena with extremely short time scales. The energy content, spectra, and angular patterns of laser-triggered ions are now being studied experimentally, theoretically, and in multi-dimensional simulations.

It has long been understood [5] that fast ion generation in a laser-produced plasma is connected with hot electrons. The commonly recognized effect responsible for ion acceleration is charge separation in a plasma due to high-energy electrons driven by a laser inside the target $[1-4,6,7]$, and/or an inductive electric field resulting in a self-generated magnetic field [8]. In addition, direct laser-ion interaction has been discussed for extremely high laser intensities of $\sim 10^{22} \mathrm{~W} / \mathrm{cm}^{2}$ [9]. High-energy electron production up to multi-MeV energies can be accomplished using several processes, such as stimulated forward Raman scattering, resonant absorption, stochastic heating, laser wakefields, ponderomotive acceleration by standing and propagating laser pulses, "vacuum heating" due to the $\mathbf{v} \times \mathbf{B}$ component of the Lorentz force or the Brunel effect; the wavebreaking of self-modulated laser wakefields, and betatron resonance provided by laser-pulse channeling. Because of the numerous mechanisms of electron gen- 
eration, many different regimes of ion acceleration are possible that hamper their corresponding investigation. At present, our main interest is investigating the mechanisms of ion acceleration in the interaction of a laser pulse with a solid target, and their dependence on the laser-pulse and plasma parameters. Clearly, a better understanding of these mechanisms and the quantification of the dependences of the ion yield in terms of the laser pulse and plasma parameters is essential for success in the new applications for laser-triggered ion beams.

Recent publications show that there is some controversy about the origin of the high-energy ions. The results obtained from some experiments [6,10-12] provide evidence that the observed $\mathrm{MeV}$-ions were generated and accelerated in plasma at the front of the target, conflicting with experiments [3, 4, 13-15], which indicated that proton acceleration took place at the back of the target. The electrostatic model of ion acceleration suggests that the origin of the ions was the same for both of these experiments and that the only difference was in the plasma thickness, i.e., whether or not the plasma extended to the rear surface. We believe both situations are possible, depending on laser pulse characteristics and foil material conductivity. Not only is there controversy about the origin of the high-energy ions but also dramatic disagreement on hot electron penetration in solid targets $[16,17]$. The latter experiments were performed using green light of approximately the same pulse-duration with laser intensities of up to $4 \times 10^{18}$ and $10^{19} \mathrm{~W} / \mathrm{cm}^{2}$, respectively. However, the penetration depth of hot electrons in [17] was more than two orders of magnitude greater than in [16]. Two possible disparate scenarios may explain these controversies in high-energy electron and ion generation:

Scenario 1. When a solid target is highly resistive, the return current within it is strongly suppressed. In this case, hot electrons generated in the laser-plasma interaction in front of the foil penetrate only to the distance of their Debye length, $\lambda_{\mathrm{Dh}}$. These electrons are pulled back within the target (if the foil thickness exceeds the Debye length of the hot electrons) by the electrostatic fields they set up, and return in the shape of a fountain. The Debye sheath accelerates the ions, which appear to originate from the front of the target.

Scenario 2. Hot electrons penetrate deep inside the foil and reach the rear of the target. Such electrons can only propagate due to the return current [18-20], which requires a high conductivity within the target. This can occur in the case of a metal target or of effective target ionization by the electrons or the electrostatic field (dielectric breakdown). Such a scenario corresponds to ion acceleration at the back of the target.

Clearly, there could be an intermediate situation between these two disparate scenarios in which the plasma has a non-monotonic density distribution within the target's thickness, even though the cold insulator might become a sort of conductor due to the laser prepulse, its ionization by fast electrons, the return current of slow electrons, and/or an electrical breakdown. The physical picture of this transformation arising in the bulk of the target is still incomplete. Note that paper [21] sheds some light on the latter question, demonstrating a fourfold difference in the penetration depth of fast electrons in metals and insulators.

This paper is aimed at reviewing the recent advances in multi-MeV ion beam generation from the interaction of short laser pulses, focused onto thin foil targets at intensities ranging from $10^{17}$ to $10^{19} \mathrm{~W} / \mathrm{cm}^{2}$, at the University of Michigan's Center for Ultrafast Optical Science (CUOS). We present our experimental results on high-energy proton generation with a 10 TW Nd:glass/Ti:sapphire laser at the fundamental and second harmonic frequencies, as well as our results on nuclear activation with protons and deuterons. The main emphasis of the presented study is the measurement of light ions, protons, and deuterons, although some results from the generation of heavy ions are also discussed. We report on the manipulation of a proton beam using shaped targets and different target material. We also present the results of our theoretical models and fully relativistic two-dimensional (2D) particle-incell (PIC) simulation of multi-MeV ion generation in the interaction of a short laser pulse with a dense plasma.

\section{ION GENERATION IN THE HIGH CONTRAST CASE}

In this set of experiments [2], the intensity contrast at the wavelength of $1.053 \mu \mathrm{m}$ was $\sim 10^{4}: 1$, as measured by the third-order correlation technique. Energy in the nanosecond prepulse due to amplified spontaneous emission at the fundamental frequency was as high as $1 \mathrm{~J}$. The contrast was improved to an estimated $10^{7}: 1$ by frequency doubling of the laser light in a 4-mm KDP crystal. The energy in green light $2 \omega_{0}$ was limited to $1 \mathrm{~J}$ because of the nonlinear distortion of the laser wavefront in the doubling crystal. The laser beam was focused on the surface of thin films of aluminum with a thickness of $1.8 \mu \mathrm{m}$ at an incidence angle of $0^{\circ}$ or $45^{\circ}$ using an $f / 3(f=16.5 \mathrm{~cm})$ off-axis parabolic mirror. The maximum focused intensity was $3 \times 10^{18} \mathrm{~W} / \mathrm{cm}^{2}$.

The high-energy ion emission was recorded by CR39 plastic nuclear track detector. To determine the maximum energy and spectrum of the ions, the detectors were covered with steps of Mylar filters having thicknesses from 2 to $50 \mu \mathrm{m}$. It was found that protons are the predominant component of a high-energy ion emission. These protons originate from a thin layer on a target surface contaminated with hydrocarbons and/or water vapor. Such target contamination was observed in the late 1960s in laser-matter interaction experiments with nanosecond laser pulses, as discussed in [5]. In our experiments, we observed a high-energy proton beam emerging through the rear surface as a beam with an 
Maximum proton energy, $\mathrm{MeV}$

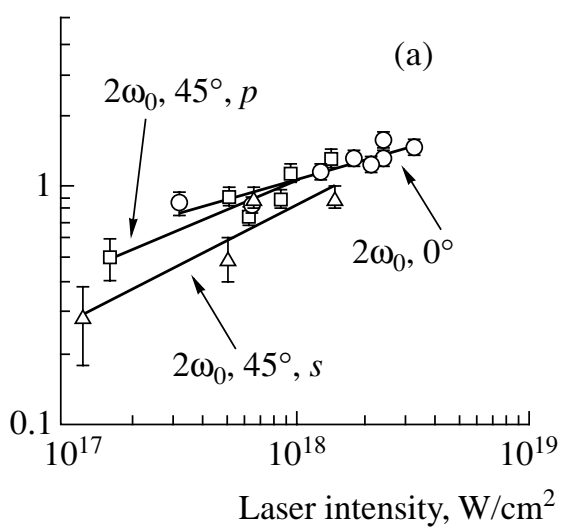

Maximum proton energy, $\mathrm{MeV}$

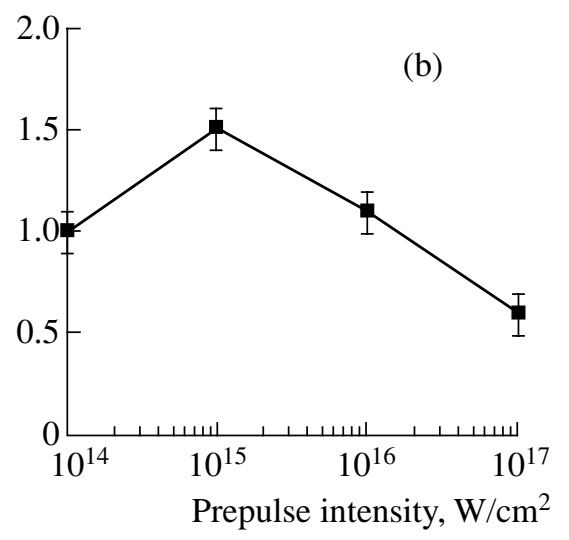

Fig. 1. Maximum proton energy in the forward direction as a function of (a) laser intensity at $2 \omega_{0}$ for different conditions of illumination of $1.8-\mu \mathrm{m} \mathrm{Al}$ foil: (circles) $0^{\circ}$; (squares) $45^{\circ}, p$-polarization; and (triangles) $45^{\circ}, s$-polarization (the solid lines are the best power function fits to the experimental data) and (b) $2 \omega_{0}$ prepulse intensity at $10^{18} \mathrm{~W} / \mathrm{cm}^{2}$ main pulse intensity (the solid line is plotted through the experimental points for better viewing).

angular divergence of about $40^{\circ} \pm 10^{\circ}$. The direction of the high energy proton beam did not depend on the angle of incidence of laser radiation and was always normal to the target surface. From this, one may conclude that the electric field of charge separation accelerating protons inside the target is directed along the target surface normal.

We studied the dependence of maximum proton energy versus laser intensity for different conditions of thin foil illumination. The highest proton energy of $1.5 \mathrm{MeV}$ was observed for normal incidence at the maximum laser intensity of $3 \times 10^{18} \mathrm{~W} / \mathrm{cm}^{2}$ (Fig. 1a). At a $45^{\circ}$ angle of incidence, the maximum intensity of the beam on the target was reduced by a factor of 2 due to the increased spot size in the horizontal direction. The maximum observed proton energy, $\epsilon_{i}^{\max }$, was comparable for both cases at the same intensity and can be fitted as the function $\epsilon_{i}^{\max } \propto I^{\alpha}$, where $\alpha$ is between 0.3 and 0.4 . Illumination with $s$-polarization at $45^{\circ}$ produced protons with energies $200-300 \mathrm{keV}$ less than those for $p$-polarization. We also observed high energy protons moving in the backward direction (in the direction of plasma expansion) for $45^{\circ}$ laser illumination, but their energy was half that of those moving in the forward direction.

We changed the plasma gradient scale length by introducing a prepulse at $2 \omega_{0}$, with a time delay of $50 \mathrm{ps}$ in front of the main $2 \omega_{0}$ pulse. The prepulse was varied with intensities ranging from $0.01 \%$ to $10 \%$ of the main pulse intensity of $10^{18} \mathrm{~W} / \mathrm{cm}^{2}$. It was found that there was an optimum prepulse intensity of about $10^{15} \mathrm{~W} / \mathrm{cm}^{2}$ (Fig. 1b) for maximum proton energy production. We estimate that at this intensity, the scale length of preformed plasma is several laser wavelengths. From this, we conclude that a preformed plasma that is not too extended enhances high-energy ion production.

The proton energy spectrum was measured by the foil attenuation method. Using steps of Mylar filters with thicknesses that differed by $2 \mu \mathrm{m}$ provided a proton energy resolution of about $100 \mathrm{keV}$. The proton spectrum in Fig. 2 shows an exponential decay from energies of $400 \mathrm{keV}$ to $1 \mathrm{MeV}$ with a characteristic temperature of $230 \mathrm{keV}$ (solid line), indicating Maxwellian-like distribution of the bulk protons. An interesting feature of the spectrum at higher energies is a plateau that ends in a sharp energy cutoff at $1.5 \mathrm{MeV}$;

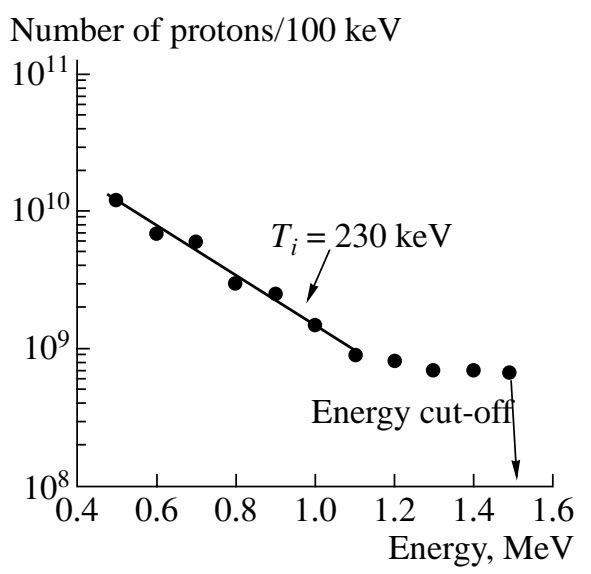

Fig. 2. Energy distribution of fast protons in the forward direction (circles), measured by attenuation of the beam in Mylar filters of different thicknesses for $2 \omega_{0}$ illumination of $1.8-\mu \mathrm{m} \mathrm{Al} \mathrm{film} \mathrm{with} \mathrm{intensity} \mathrm{of} 2 \times 10^{18} \mathrm{~W} / \mathrm{cm}^{2}$ at normal incidence. The arrow shows the sharp cutoff in proton energy at a spectral distribution below the detection threshold. 


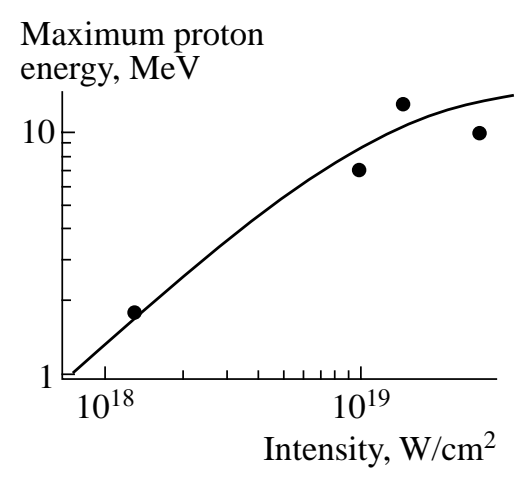

Fig. 3. Maximum proton energy dependence (dots), measured by attenuation of the beam in Mylar filters of different thicknesses for the illumination of a $10-\mu \mathrm{m} \mathrm{Al}$ film with laser pulse of $1.053 \mu \mathrm{m}$ wavelength at a $45^{\circ}$ angle of incidence.

this is typical of an electrostatic mechanism of ion acceleration.

\section{ION GENERATION \\ IN THE LOW CONTRAST CASE}

These experiments were performed at the fundamental wavelength of $1.053 \mu \mathrm{m}$ with an intensity contrast of $5 \times 10^{5}: 1$. The $p$-polarized laser beam was focused on the surface of Mylar or aluminum foils of different thickness at a $45^{\circ}$ angle of incidence. Maximum laser intensity on the target was $\approx 2 \times 10^{19} \mathrm{~W} / \mathrm{cm}^{2}$. Aluminum targets typically produced proton beams with energies almost twice as high as Mylar targets. The highest energies for aluminum and Mylar targets were 13 and $7 \mathrm{MeV}$, respectively. The maximum proton energy for aluminum foil was about three times higher

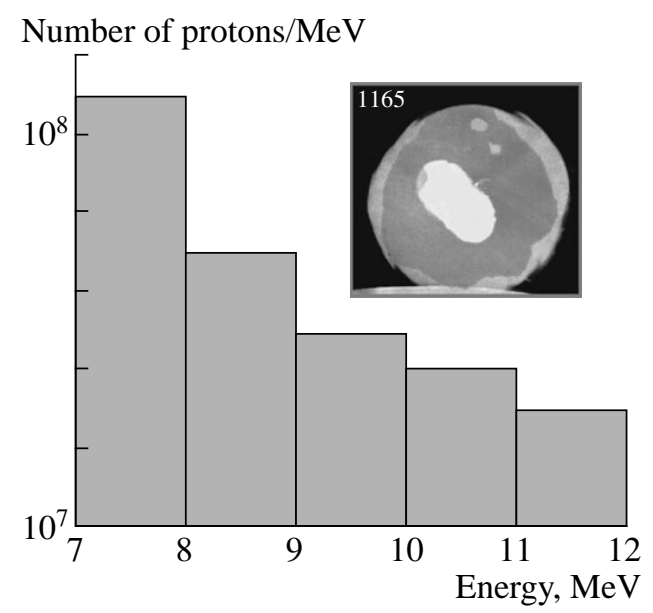

Fig. 4. Spectrum of proton beam from a $12.5-\mu \mathrm{m}$ aluminum target at a laser intensity of $\sim 10^{19} \mathrm{~W} / \mathrm{cm}^{2}$, received by track counting in the CR-39 detector. The inset shows a forward accelerated proton beam with energy above $4 \mathrm{MeV}$. than in the high contrast case (Section 2) for the same laser intensities. This confirms that preplasma enhances high-energy ion production: at an intensity contrast of $5 \times 10^{5}: 1$, preplasma very likely appears before the maximum laser energy reaches the target.

We studied for the first time the dependence of maximum proton energy as a function of laser intensity for an aluminum target and found that this dependence was quite strong at $1.5 \times 10^{18}<I<10^{19} \mathrm{~W} / \mathrm{cm}^{2}$, quite close to $\propto I$, with a tendency to saturate at higher intensities. This behavior of the maximum proton energy is illustrated in Fig. 3. The maximum proton energy for a Mylar target at $I>4 \times 10^{18} \mathrm{~W} / \mathrm{cm}^{2}$ was also close to a $\sqrt{I}$-dependence. Qualitatively, the shape of the proton energy spectrum is similar to that observed with the high contrast laser, i.e. it is characterized by a plateau at the high end of the energy spectrum which typically has a sharp cutoff. Figure 4 presents the high-energy portion of a proton spectrum from an aluminum target. From this spectrum we can estimate that the number of protons with energy higher than $10 \mathrm{MeV}$ is about $10^{8}$. The inset of Fig. 4 shows an image of the proton beam with energy above $4 \mathrm{MeV}$. The bright elliptical region within the beam corresponds to protons that passed through $1 \mathrm{~mm}$ of CR-39 and thus left tracks on the rear surface of the detector. This imprint corresponds to protons with energies above $12 \mathrm{MeV}$.

Some measurements demonstrated that proton spectra from Al target may have a band structure, as shown in Fig. 5. Note that the efficiency of high-energy proton production is very sensitive to the contrast of the laser pulse. Proper laser plasma matching at the front of a target helps to achieve extreme parameters, while the production of a long preplasma significantly decreases the maximum proton energy. Figure 5, for instance, corre-

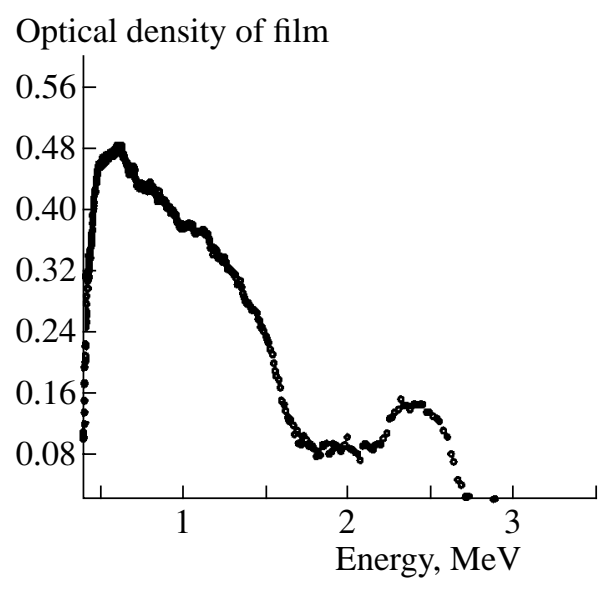

Fig. 5. Proton spectrum showing the band structure from the interaction of an $12.5-\mu \mathrm{m}$ Al film with a laser pulse of $1.053-\mu \mathrm{m}$ wavelength and intensity $5 \times 10^{18} \mathrm{~W} / \mathrm{cm}^{2}$ at a $45^{\circ}$ angle of incidence. The spectrum was photographed using a dipole magnetic spectrometer and X-ray film. 

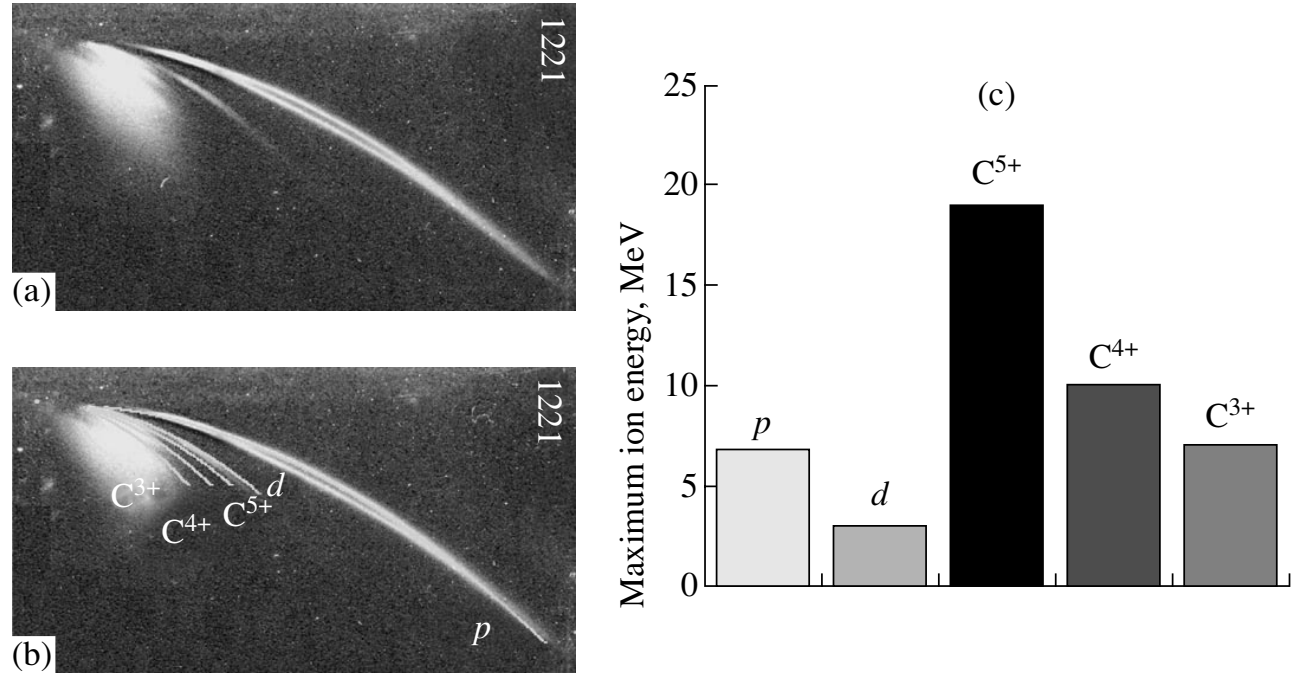

Fig. 6. (a) Ion traces obtained with a Thomson parabola spectrometer from a front deuterated Mylar target irradiated with a laser intensity of $\sim 10^{19} \mathrm{~W} / \mathrm{cm}^{2}$. (b) The same as in plot (a) with ion identification (parabolas are added to better follow the traces). (c) Distribution of maximum ion energy for different ion species.

sponds to less than optimal laser contrast conditions $\left(\sim 10^{4}: 1\right)$, so that the maximum proton energy is less than $3 \mathrm{MeV}$ for a laser intensity of $\sim 5 \times 10^{18} \mathrm{~W} / \mathrm{cm}^{2}$.

Using a Thomson parabola spectrometer with parallel electric and magnetic fields $(B=0.26 \mathrm{~T}, E=$ $7.7 \mathrm{kV} / \mathrm{cm}$ ) in an experiment with a front deuterated Mylar target, we registered proton and heavier ion emission in the forward direction in a single laser shot. Figure 6a presents an image of a CR-39 plate that was used for ion detection. We identified traces of protons, deuterons, and carbon ions: $\mathrm{C}^{5+}, \mathrm{C}^{4+}$, and $\mathrm{C}^{3+}$ (Fig. 6b). The maximum ion energies were measured from these traces. Figure $6 \mathrm{c}$ shows the maximum energies for different ion species. We found that the maximum energies for different ion species were as follow: $\approx 7 \mathrm{MeV}$ for protons, $\approx 2.7 \mathrm{MeV}$ for deuterons, $\approx 19 \mathrm{MeV}$ for $\mathrm{C}^{5+}$, $\approx 10 \mathrm{MeV}$ for $\mathrm{C}^{4+}$, and $\approx 7 \mathrm{MeV}$ for $\mathrm{C}^{3+}$. This corresponds to energies per nucleon of $\approx 7 \mathrm{MeV}$ for protons, $\approx 1.35 \mathrm{MeV}$ for deuterons, $\approx 1.6 \mathrm{MeV}$ for $\mathrm{C}^{5+}, \approx 0.8 \mathrm{MeV}$ for $\mathrm{C}^{4+}$, and $\approx 0.6 \mathrm{MeV}$ for $\mathrm{C}^{3+}$. This experiment provides evidence of the possibility of accelerating light ions more efficiently than heavier ions. We prove below that the protons in our experiment were accelerated predominantly from the front of the target, as in [1], while it was asserted in $[3,4]$ that proton acceleration came from the back of the target.

Heavier ions are more likely to be accelerated at the back of the target [22] because of high losses within the foil. We found that the $\mathrm{C}^{5+}$ trace was very weak, compared to the $\mathrm{C}^{4+}$ and $\mathrm{C}^{3+}$ traces. This means that a very small number of $\mathrm{C}^{5+}$ ions were created at the back of the target. The ionization at the back of the target was caused by the electrostatic field created by high-energy electrons penetratingthrough it. This field not only ion- izes atoms but accelerates ions to high energies. In a similar experiment on a 6- $\mu \mathrm{m}$ Mylar target without a deuterated layer, no trace of $\mathrm{C}^{6+}$ ions was observed. From the fact that $\mathrm{C}^{4+}$ ions are produced in abundance and $\mathrm{C}^{5+}$ generation is very weak, one may find the strength of the electrostatic field at the back of the target using the Ammosov-Delone-Krainov model [23]

$$
E \geq(2 / 3)\left(U_{4} / U_{\mathrm{H}}\right)^{3 / 2} E_{a},
$$

where $E_{a}=0.51 \times 10^{10} \mathrm{~V} / \mathrm{cm}$ is the atomic electric field, and $U_{N}(N=1,2, \ldots)$ and $U_{\mathrm{H}}$ are the ionization potentials of the ionized species and hydrogen, respectively. From Eq. (1) we find $E \approx(3-4) \times 10^{10} \mathrm{~V} / \mathrm{cm}$.

\section{NUCLEAR ACTIVATION WITH DEUTERONS AND PROTONS}

In our experiments at the fundamental frequency [10] with a maximum intensity of $\approx 6 \times 10^{18} \mathrm{~W} / \mathrm{cm}^{2}$ and an intensity contrast of $5 \times 10^{5}: 1$, we carried out the activation of a sample of boron isotope ${ }^{10} \mathrm{~B}$. The highintensity laser beam was focused on a Mylar target covered by a deuterated polystyrene layer. The resultant ion beam (protons and deuterons) was used to irradiate an enriched sample of ${ }^{10} \mathrm{~B}$ (enrichment $90 \%$ ). A cylindrically-shaped sample $10 \mathrm{~mm}$ in diameter and $5 \mathrm{~mm}$ in thickness was positioned on the CR-39 detector with a few steps of Mylar filters to monitor the energy of the ion beam and its alignment. The deuterated polystyrene layer, $\geq 1 \mu \mathrm{m}$ thick, was deposited on the front side of the 6- $\mu \mathrm{m}$ Mylar film. The experimental setup is shown in Fig. 7 a. 
(a)

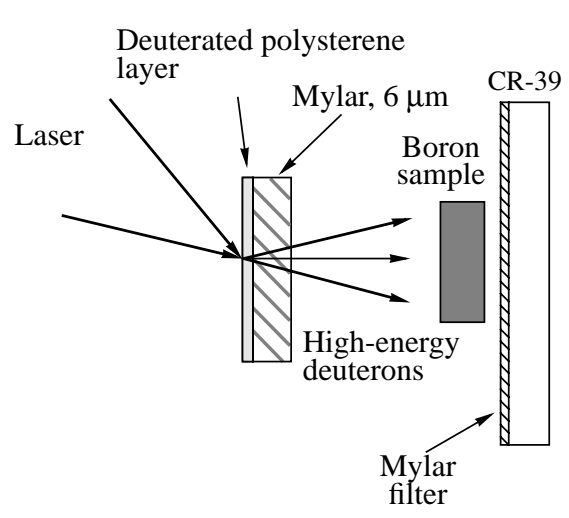

(b)

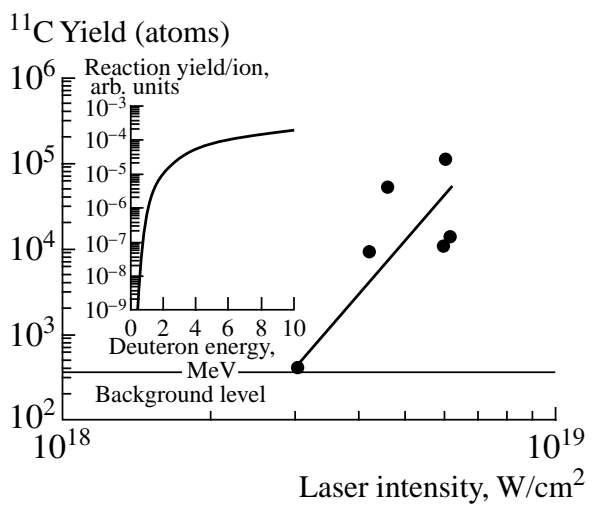

Fig. 7. Schematic diagram of boron sample activation with high-energy deuterons (a). Experimental yield of ${ }^{11} \mathrm{C}$ versus the laser intensity for the ${ }^{10} \mathrm{~B}(d, n){ }^{11} \mathrm{C}$ reaction (b). The inset shows calculated yield of ${ }^{11} \mathrm{C}$ per one deuteron as a function of the energy cutoff for a uniform energy distribution of deuterons.

The concentration of boron isotope ${ }^{10} \mathrm{~B}$ in natural boron is about $20 \%$. Therefore, in the enriched boron sample used, the yield of isotope ${ }^{11} \mathrm{C}$ in the reaction ${ }^{10} \mathrm{~B}(d, n){ }^{11} \mathrm{C}$ must be enhanced approximately by a factor of 4.5 , as compared with the $(p, n)$ reaction. The yield of the $\beta^{+}$-active ${ }^{11} \mathrm{C}$ was measured by counting the number of positrons emitted. The coincidence measurements require a low background count rate in order to obtain a decent signal to noise ratio. Two 3 -inch $\mathrm{NaI}$ detectors placed opposite one another were used to detect the coincidence signal resulting from the annihilation of electrons and positrons and the production of two gamma photons with an energy of $0.511 \mathrm{MeV}$. The detection efficiency was measured as being in the range of $0.05-0.10$ using a ${ }^{22} \mathrm{Na}$ radioactive source with a precisely known activity of positron emission. The background noise was found to be 1-5 count/min, while the radioactivity detection threshold was $\sim 10 \mathrm{pCi}$.

We identified the source of this coincidence signal as the positron decay of the carbon isotope ${ }^{11} \mathrm{C}$, which has a half-life of $20.4 \mathrm{~min}$. We measured a maximum of $\approx 300$ counts/min at $330 \mathrm{~s}$ after the laser shot. The maximum total yield of ${ }^{11} \mathrm{C}$ is estimated to be $\sim 10^{5}$; this corresponds to $\approx 2 \mathrm{nCi}$ of radioactivity immediately after the shot. The laser was able to deliver shots with a time interval of approximately $7 \mathrm{~min}$. Therefore, the accumulated effect of several shots will increase radioactivity only by a factor of 3 . If a similar laser could operate with a $10-\mathrm{Hz}$ repetition rate, a positron source with an activity of $\approx 20 \mu \mathrm{Ci}$ could be built up if the irradiation time were equal to the half-life of ${ }^{11} \mathrm{C}$.

We observed a very sharp power dependence of the ${ }^{11} \mathrm{C}$ yield as a function of laser intensity (Fig. 7b), indicating that deuteron energies are near the threshold for the ${ }^{10} \mathrm{~B}(d, n){ }^{11} \mathrm{C}$ reaction, $\simeq 2 \mathrm{MeV}$. In the same set of experiments, we measured the maximum proton energy at about $5 \mathrm{MeV}$ at the highest laser intensity. The much lower deuteron energies confirm the electrostatic mechanism of ion acceleration. Deuterons are accelerated less because they are two times heavier than protons and have less mobility and higher energy losses. Since they follow behind the proton bunch, they experience a significantly smaller accelerating electric field. Consequently, they would be expected to have a lower energy than protons.

To verify that deuteron acceleration is more efficient from the front side of the target, we irradiated a $6-\mu \mathrm{m}-$ thick Mylar target with a layer of a deuterated plastic on its back side, and performed the same ${ }^{10} \mathrm{~B}$ activation experiment described above. In this case, the activation signal was not above the background level. These results indicate unequivocally that, for a dielectric target at the laser intensity and intensity contrast ratio used, deuterons are either accelerated mainly from the front side of the foil, or they acquire higher energy there, compared with that from the rear side. We relate both cases to front side ion generation.

High-energy protons may participate in the ${ }^{11} \mathrm{~B}(p, n){ }^{11} \mathrm{C}$ reaction, with the cross section of this reaction [24] having a higher threshold (above $3 \mathrm{MeV}$ ). Taking into account that the amount of ${ }^{11} \mathrm{~B}$ in boron sample was only $10 \%$, we may expect the ${ }^{11} \mathrm{C}$ yield for this reaction to be much less than that for deuterons. To verify that the $(p, n)$ reaction does not contribute substantially to the ${ }^{11} \mathrm{C}$ yield, we focused the laser at its highest intensity on a bare Mylar film, behind which was a boron sample. In this case, no activation signal was observed.

Our experiments with aluminum targets showed that the maximum proton energy (Fig. 3) can exceed severalfold the threshold for some $(p, n)$ reactions. Guided by this, we performed an experiment on the nuclear activation of ${ }^{11} \mathrm{~B}$ and ${ }^{63} \mathrm{Cu}$ samples. The thresholds for these reactions were 3 and $4 \mathrm{MeV}$, while the maxima of the reaction cross-sections were relatively 


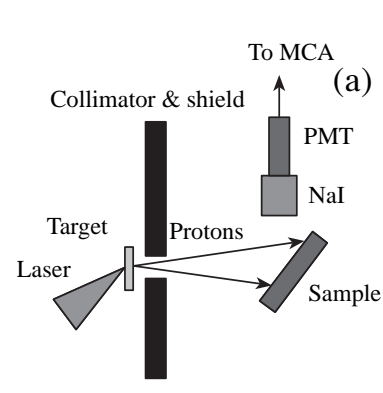

Counts $(0.511 \mathrm{MeV})$

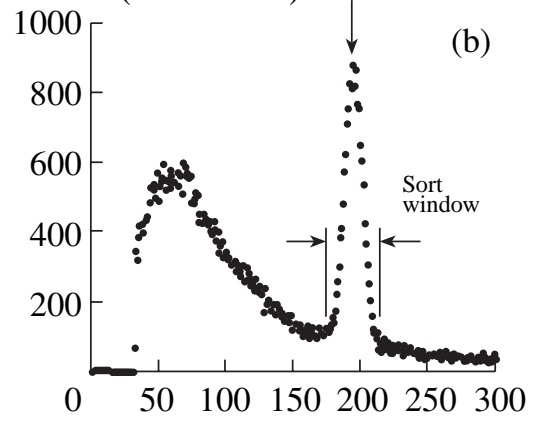

Channel

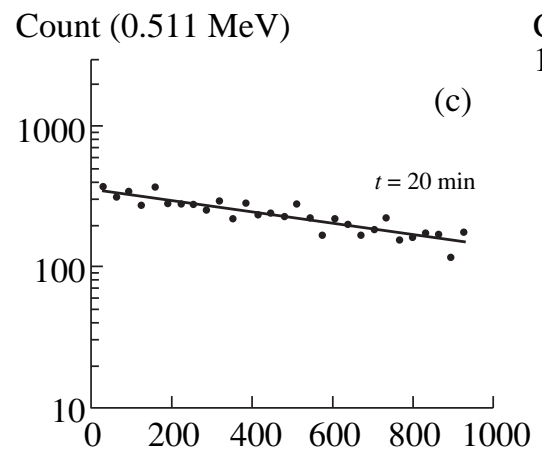

Count $(0.511 \mathrm{MeV})$

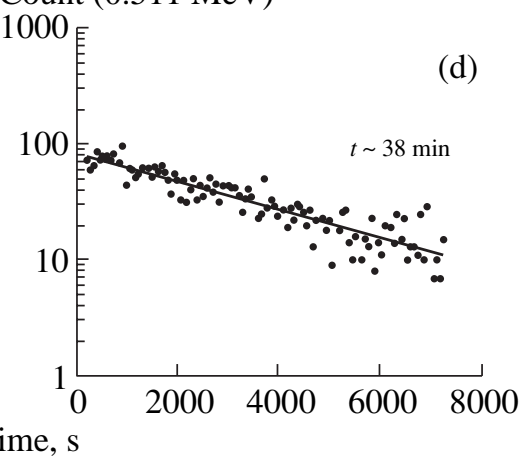

Fig. 8. Setup for activation with protons. Collimator and shield were introduced to prevent saturation of the detection system due to high-energy electrons and gamma photons during the laser shot (a). Spectrum of proton irradiated ${ }^{11} \mathrm{~B}$ sample (b). Radioactive decay signals (c and d) of proton irradiated ${ }^{11} \mathrm{~B}$ and ${ }^{63} \mathrm{Cu}$ samples (dots). Solid lines correspond to the decay of ${ }^{11} \mathrm{C}$ and ${ }^{63} \mathrm{Zn}$ with half-life of 20 and 38 min, respectively.

high: $\simeq 400$ and $500 \mathrm{mb}$, respectively. The products of these reactions were ${ }^{11} \mathrm{C}$ and ${ }^{63} \mathrm{Zn}$, which are positron emitters with half-lives of 20 and 38 min, respectively. A schematic of the experimental setup is shown in Fig. 8a. Measurements of the induced radioactivity were performed in situ in the interaction chamber. A laser-triggered, high-energy proton beam irradiated a sample placed a few centimeters behind the target. The emission spectrum of the sample was monitored by a $\mathrm{NaI}$ detector coupled to a photomultiplier tube (PMT), with readout performed by a multi-channel analyzer (MCA). Figure 8b shows the most significant part of the ${ }^{11} \mathrm{C}$ spectrum, plotted on a vertical linear scale. A peak is clearly seen between spectral channels 180 to 220; this corresponds to the annihilation of positrons and electrons and the production of gamma photons with an energy of $0.511 \mathrm{MeV}$. The points at the lowest channel numbers are zero because the $\mathrm{NaI}$ signal was below the discriminator value set on the analog-to-digital converter. The broad peak well below $0.511 \mathrm{MeV}$ is due to Compton scattered electrons. The gated region in Fig. $8 \mathrm{~b}$ defines the counts that were included in the resorted signal as a function of time, thus yielding the half-life for ${ }^{11} \mathrm{C}$. Figures $8 \mathrm{c}$ and $8 \mathrm{~d}$ present experimental radioactive decay signals for proton irradiated ${ }^{11} \mathrm{~B}$ and ${ }^{63} \mathrm{Cu}$ samples; the signals show the production of $\sim 10^{5}$ atoms of ${ }^{11} \mathrm{C}$ and ${ }^{63} \mathrm{Zn}$.

\section{PROTON BEAM CHARACTERISTICS AND MANIPULATION}

The manipulation of the ion beam characteristics is an important part of the problem under discussion. From the theoretical point of view, some ideas for the manipulation of ion beams have already been proposed, e.g., in [8, 25-28]. However, these methods require great precision in the manufacturing and control of targets (features on the order of a micron, positioned with submicron accuracy at the laser focus), which is far from trivial. We carried out experiments on beam profile manipulation by modest modification of the target geometry, thereby extending the concept first proposed in [8] of tailoring a target foil and demonstrating the promise of this method.

At multi-MeV energies, the protons originating in laser-irradiated thin foils are formed into a well-collimated beam. Even at an energy of $\sim 1 \mathrm{MeV}$, they can be confined in a quite narrow cone. Figure 9 shows a typical image of the proton beam in the forward direction (behind the target) observed in our experiment with $2 \omega_{0}$ irradiation of a thin aluminum foil. A collimated beam of fast protons is observed, with energies as high as $1.5 \mathrm{MeV}$ and total number of $\geq 10^{9}$, confined in a cone angle of $40^{\circ} \pm 10^{\circ}$. 


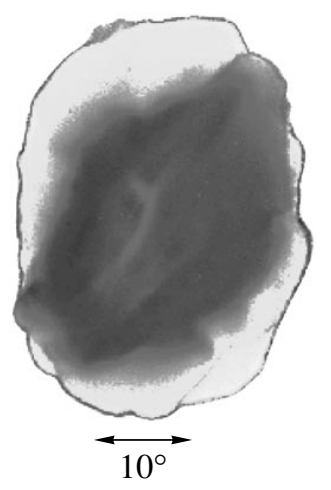

Fig. 9. Typical image of a proton beam observed traveling in the forward direction from the interaction of a high-intensity laser with a $1.8-\mu \mathrm{m}$-thick aluminum foil. The proton beam passed through a $25-\mu \mathrm{m}$ Mylar filter, corresponding to an energy above $1.2 \mathrm{MeV}$. The laser intensity on the target is $2 \times 10^{18} \mathrm{~W} / \mathrm{cm}^{2}$ at $2 \omega_{0}$ illumination normal to the target.

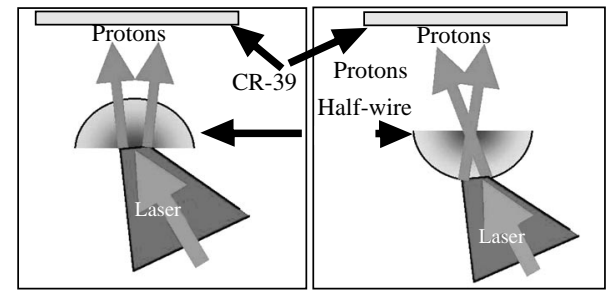

(a) Flat

(b) Round

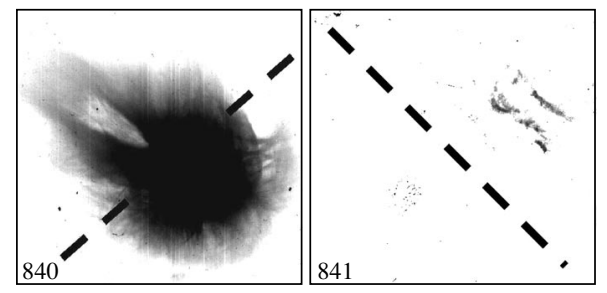

Fig. 10. Scheme of the irradiation of a $100-$ to $120-\mu \mathrm{m}-$ diameter aluminum half-wire target. The protons from the flat (a) and round (b) sides of the irradiated target are recorded using the CR-39 detector. The wires were oriented approximately $45^{\circ}$ to the vertical, as shown by dashed lines.

Using an aluminum half-wire target profile between $100-120 \mu \mathrm{m}$, we observed an effect analogous to that of an electrostatic cylindrical lens. The laser was focused onto either the flat or rounded surface of the half-wire, and the beam image was recorded. A CR-39 detector was used to record the protons. It was placed approximately $2 \mathrm{~cm}$ from the target (Fig. 10). The irradiation of the flat surface of a target having a cylindrical rear surface and vice versa is shown in Fig. 10a and Fig. 10b, respectively. A target with a flat front side surface of the half-wire produces a proton beam similar to that of an ordinary film target. When a round surface is irradiated, an uncollimated spray of protons from the flat side of the half-wire appears, such as one might expect from a cylindrical lens. The results of these experiments may support studies showing front side proton acceleration.

An important question which must addressed for the future application of laser-triggered protons is the possibility of focusing the proton beam. To investigate this, we performed experiments with shaped (concave) $25-\mu \mathrm{m}$ aluminum foil targets. The CR-39 detector was placed $3 \mathrm{~cm}$ behind the target. We observed an oversaturated region in the center of the proton beam. This region appeared brighter due to a large number of overlapping tracks scattering more light. Physical inspection of this region under a microscope revealed the higher track density, differentiating it from regions of similar opacity but with lower track density. One might think that the ballistic focusing of a proton beam can be achieved only at the plane passing through the center of the target curvature. Indeed, this would be true if the proton beam emerging from a flat target were collimated. In reality, a laser-triggered proton beam has quite a large divergence; moreover, protons of different energies have different divergences. Typically, the higher energy proton component has a narrower cone. This may explain our observation of the focused part of the beam seen in Fig. 11 at distances much greater than the radius of curvature of the shaped target. According to our estimates, this focused region may correspond to the high-energy proton beam component, which would have a divergence of about $5^{\circ}$ if a flat target were used.

Along with the difference in proton energies (Section 3), we observed a significant difference in the proton beam quality for metal (Al) and dielectric (Mylar) targets. In Figs. 12 and 13, the proton beam quality is illustrated for different proton energies. Generally, aluminum targets produce higher quality beams with more uniform proton beam profiles. In contrast, the proton beams from Mylar show significant inhomogeneity. We also found that, for both types of targets, the lower energy component of the proton beam is more structured then the higher energy component. One may imagine that the electric field that accelerates ions is more homogeneous in a conductive media, because of the presence of free electrons that provide a return current inside the target, which produces a more uniform proton beam.

Coating the front surface of a target with materials that provide a high absorption of laser light can significantly enhance the generation of hot electrons.In turn, this should lead to an increase in the efficiency of highenergy ion generation. We performed such an experiment with a so-called "laser black" target (a foil coated by soot-like substance) by coating $10 \mu \mathrm{m}$-thick $\mathrm{Cu}$ target with 5-10 $\mu \mathrm{m}$-thick laser black, structured with a characteristic scale of 1-2 $\mu \mathrm{m}$. The absorption in such a target is practically ideal $(>96 \%)$. When laser light was focused into a spot $6-\mu \mathrm{m}$ in diameter, we observed 


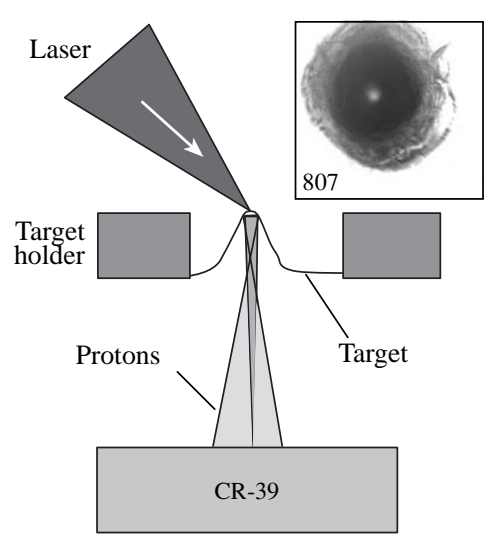

Fig. 11. Scheme of the irradiation of a curved target. Inset presents the proton beam image on the CR-39 for an energy greater than $2 \mathrm{MeV}$.

a doubling of the maximum ion energy $(8.2 \mathrm{MeV})$, compared to a target without coating $(4 \mathrm{MeV})$. The increase in ion energy was not followed by a reduction in proton beam quality (Fig. 14).

Borghesi et al. showed that point projection proton imaging [29] appears to be a powerful technique for electric field detection in laser-irradiated targets and plasmas $[30,31]$. This technique was further developed by Mackinnon and coworkers [32,33] to produce Moire fringes in a proton beam. We performed a preliminary experiment on imaging with high-energy proton beams. A free-standing copper mesh with a wire diameter of $10 \mu \mathrm{m}$ and a period of $30 \mu \mathrm{m}$ was illuminated by a proton beam produced in the interaction of the high-intensity laser with a thin film target. The mesh was parallel to the thin film target (Fig. 15a). The distance between the proton source and the mesh was $5 \mathrm{~mm}$, while the radiochromic film was positioned at $50 \mathrm{~mm}$ from the source, thus providing a tenfold magnification. A shadow of the mesh was observed on the radiochromic film, as shown in Fig. 15b. The optical density modulation on the film was of the order of 0.2 . It must be noted that the stopping range of $10-\mathrm{MeV}$ pro- tons in $\mathrm{Cu}$ is about $400 \mu \mathrm{m}$. Such energetic protons will lose only $0.1 \%$ of their energy in $10 \mu \mathrm{m}$ of $\mathrm{Cu}$. Such variation in energy loss across the wire was too small to produce any visible effect using our diagnostics. The current explanation of the observed phenomena is based on the assumption of a point-like proton source with very low transverse emittance, which leads to the possibility of detecting the small angle scattering experienced by protons inside a thin wire. Proton imaging can be very useful for the diagnostics of dense plasmas, and may be used to study the distribution of electric and magnetic fields in plasmas of laser-irradiated targets with high temporal and spatial resolution [29]. Unique advantages of a laser-triggered proton source are ultrashort pulse duration and absolute synchronization for use in pump-probe experiments; in addition, the pointlike source provides a spatial resolution of several microns for imaging applications. Other advantages are the directionality and high brightness of proton beams, while the long stopping range of $10-$ to $15-\mathrm{MeV}$ protons allows them to penetrate $\sim 1$-mm-thick objects.

\section{ION ACCELERATION BY HOT ELECTRONS}

A brief picture of ion acceleration in short-laserpulse interaction with foils was proposed in $[3,4,10$, 34]. Electrons, accelerated in an underdense plasma (with density less than critical, $n_{\mathrm{cr}}$ ), penetrate deep inside the target or even through it, setting up a strong electrostatic field (a Debye sheath) which accelerates ions forward and decelerates the electrons. Thus, during plasma expansion, the kinetic energy of the fast electrons is transformed into the energy of the electrostatic field, which accelerates the ions; their energy is expected to be on the level of the hot-electron energy. Similar physics were been explored in ICF studies of plasma expansion [35-42] with cold background electrons and laser heated electrons, which accelerate ions [5]. Though the key idea of using a two-electron-temperature model is the same, the typical hot-to-cold-temperature ratio is very different for the values of ICF and short laser-pulse produced plasmas. In an ICF plasma,

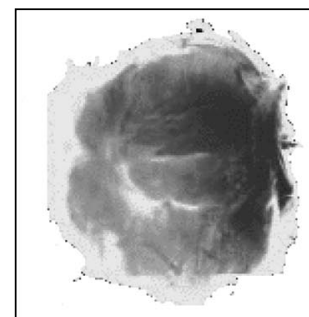

(a)

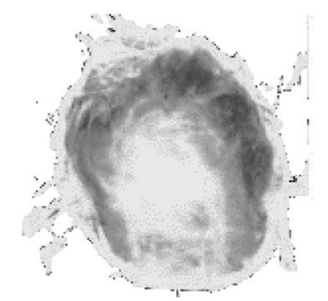

(b)

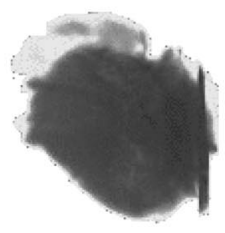

(c)

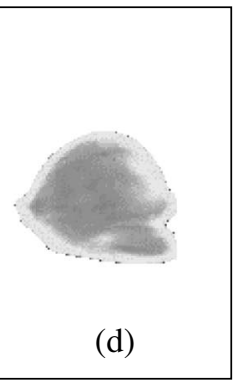

(d)

Fig. 12. Beam images on the CR-39 from a $12.5-\mu \mathrm{m}$-thick aluminum target showing the beam energy increase along the axis. Each image was taken from separate shots of similar energies with filters aimed to block protons below (a) 2.5 , (b) 3 , (c) 4.8 , and (d) $7.9 \mathrm{MeV}$. 


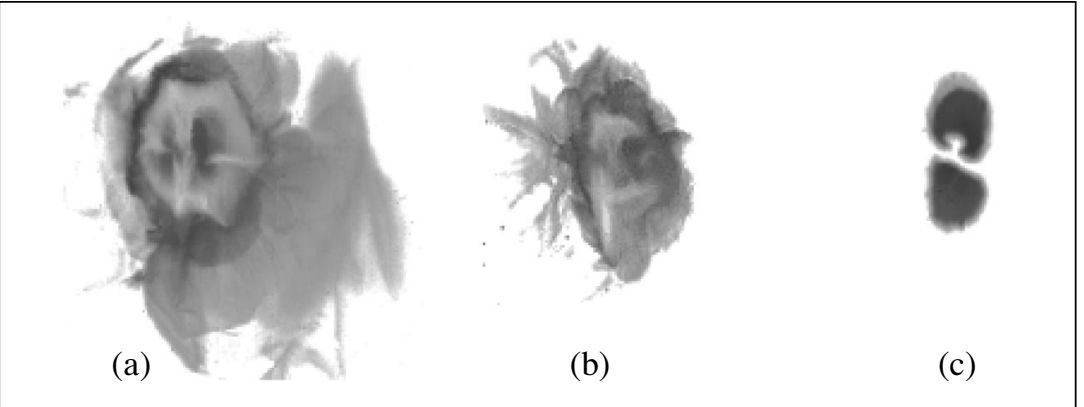

Fig. 13. Beam images on the CR-39 from a 13- $\mu$ m-thick Mylar target corresponding to proton energies above (a) 1.4 , (b) 2 , and (c) $2.7 \mathrm{MeV}$.
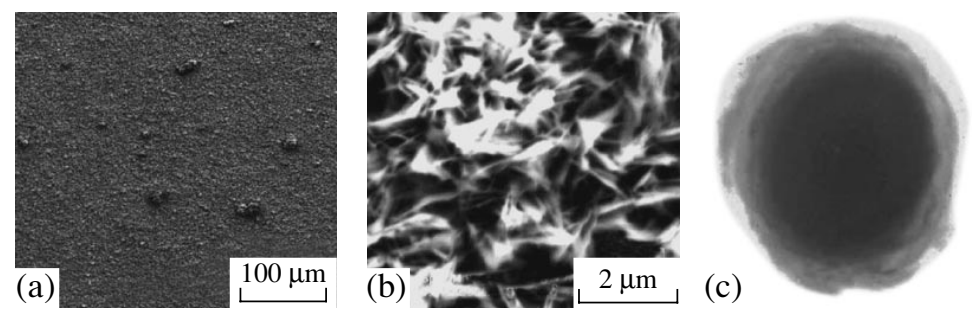

Fig. 14. (a) Low and (b) high magnification image of the surface of a "laser-black" copper target; (c) proton beam image on the CR-39 from "laser black" copper target.

(a)

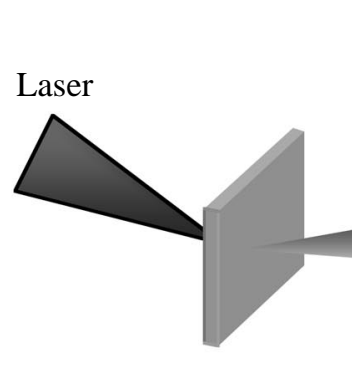

Thin film (target)

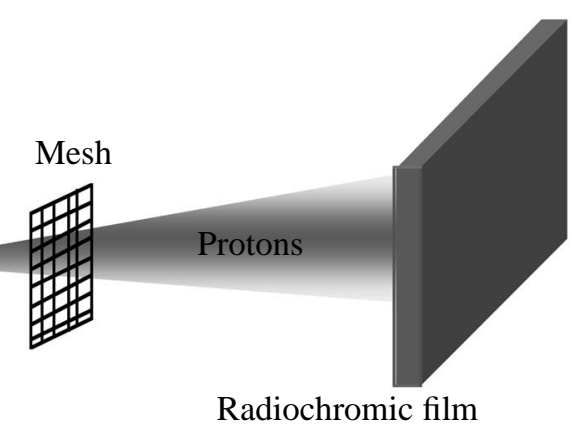

(b)

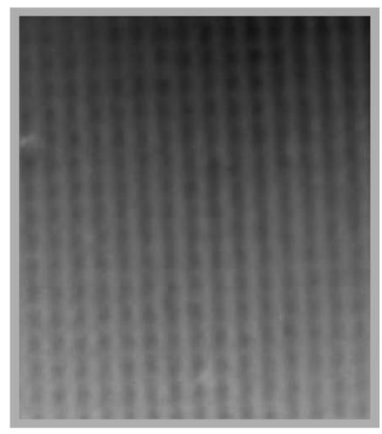

Fig. 15. (a) Setup for proton imaging of a mesh; (b) image of a mesh with $30-\mu \mathrm{m}$ period $(10-\mu \mathrm{m} \mathrm{Cu}$ wires) obtained with highenergy protons.

this ratio is usually less than $10^{2}$, while it is $\geq 10^{3}$ for current experiments on short laser-pulse interaction with a plasma. Given that most theoretical works on ion generation include numerical calculations, such a dramatic gap between hot and cold electron temperatures requires more accurate modeling to quantify this effect.

Using certain simplified theoretical models, plasma expansion into a vacuum has long been extensively studied, ever since the work by Gurevich et al. [43]. Until the last decade, however, this problem was treated using hydrodynamic models only [44]. By the 1990s, the kinetic aspects of plasma expansion had already prevailed. The latest developments in the kinetic theory of plasma expansion have brought analytical results from ionacceleration [45-47]. Certainly, PIC simulations are free from such simplification and constitute a powerful tool for studying ion acceleration in short laser-pulse interactions with plasmas starting from the basic principles. Nevertheless, PIC models are quite demanding, and some physical aspects are often hidden among the numerous interfering effects included in such cumbersome simulations. This is why it is also 
useful to explore simpler models which are still able to qualitatively describe the main features of ion acceleration and its characteristics in terms of plasma and laser parameters. This may provide an easy-to-use tool for the analysis of experimental data.

Considerable progress in the analytical description of ion acceleration by hot electrons has been achieved using the model of quasineutral plasma expansion. One may find this model to be oversimplified for interpreting the experiments on ion acceleration in expanding plasmas heated by ultra short laser pulses. However, it is definitely useful at long time scales (which are inaccessible to full-scale PIC simulations), when the laser pulse terminates and the plasma trends toward quasineutrality but acceleration continues. We start here from this model $[46,47]$.

Using the renormalization group symmetry (RGS) method in [48], it is now possible to apply the quasineutral plasma expansion model to ion acceleration. As an example, we consider a symmetrical plasma slab consisting of two ion $(\mathrm{C}$ and $\mathrm{H})$ and two electron (cold and hot) species with density profiles following the quasineutrality condition, and use the RGS method to solve the Cauchy problem for the Vlasov equations:

$$
\begin{gathered}
\partial_{t} f^{\alpha}+v \partial_{x} f^{\alpha}-\left(e_{\alpha} / m_{\alpha}\right)\left(\partial_{x} \Phi\right) \partial_{v} f^{\alpha}=0, \\
\left.f^{\alpha}\right|_{t=0}=f_{0}^{\alpha}(x, v) .
\end{gathered}
$$

Particle distribution functions $f^{\alpha}(t, x, v)$ for the electrons $(\alpha=\mathrm{c}$ and $\mathrm{h}$ for cold and hot electrons, respectively) and ions ( $\alpha=1$ and 2 for heavy and light ions, respectively) are assumed to satisfy the quasineutrality condition:

$$
\int d v \sum_{\alpha} e_{\alpha} f^{\alpha}=0, \quad \int d v v \sum_{\alpha} e_{\alpha} f^{\alpha}=0
$$

The electric potential is expressed in terms of the moments of the distribution functions:

$$
\partial_{x} \Phi=-\int d v \sum_{\alpha} e_{\alpha} v^{2} \partial_{x} f^{\alpha}\left\{\int d v \sum_{\alpha} \frac{e_{\alpha}^{2}}{m_{\alpha}} f^{\alpha}\right\}^{-1} .
$$

The key is to find the RGS that provides an invariance for the solution to the initial value problem for $t \longrightarrow 0: f^{\alpha}=\mathscr{F}^{\alpha}(t, x, v) \equiv f_{0}^{\alpha}(x, v)+O(t)$ and to formulate the finite transformations that extend this solution to the solution for $t>0$. Correspondingly, the RGS can be found as a subgroup of the group of point Lie transformations described by Eqs. (2) and (3). For the spatially symmetric initial distribution functions, the RGS generator appears as a linear combination of generators of projective transformations and translations along $t$. The finite RGS transformation defines a trans- formation of the phase space $\left(x^{\prime}, v^{\prime}\right)$ at $t=0$ to the phase space $(x, v)$ at $t>0$ as follows

$$
x^{\prime}=\frac{x}{\sqrt{1+\Omega^{2} t^{2}}}, \quad v^{\prime 2}+\Omega^{2} x^{\prime 2}=v^{2}+\Omega^{2}(x-v t)^{2},
$$

where the characteristic time scale $\Omega^{-1}=L_{0} / c_{s}$ can be treated physically as the ratio of the plasma slab halfthickness $L_{0}$ to the characteristic ion acoustic velocity, defined by the main ion component, $c_{s}=$ $\sqrt{\left(T_{1}+Z T_{\mathrm{c}}\right) /\left(m_{1}+Z_{1} m_{e}\right)}$.

The distribution functions are invariants of the group transformations (5),

$$
f^{\alpha}=f_{0}^{\alpha}\left(I^{(\alpha)}\right), \quad I^{(\alpha)}=\frac{1}{2}\left(v^{\prime 2}+\Omega^{2} x^{\prime 2}\right)+\frac{e_{\alpha}}{m_{\alpha}} \Phi_{0}\left(x^{\prime}\right),
$$

so that the distribution functions at the given time, $t \neq 0$, can be expressed in terms of their initial values. Here, the dependence of $\Phi_{0}$ on self-similar variable $x^{\prime}=$ $x / \sqrt{1+\Omega^{2} t^{2}}$ is defined by the quasineutrality condition (3), and the electric potential is given by

$$
\Phi(t, x)=\Phi_{0}\left(x^{\prime}\right)\left(1+\Omega^{2} t^{2}\right)^{-1} .
$$

We assume that initially $(t=0)$, the ion species have Maxwellian distribution functions with densities $n_{10}$, $n_{20}$ and temperatures $T_{1}, T_{2}$, and the electrons obey a two-temperature Maxwellian distribution function with densities and temperatures of the cold and hot components $n_{\mathrm{c} 0}$ and $n_{\mathrm{h} 0}\left(n_{\mathrm{c} 0}+n_{\mathrm{h} 0}=Z_{1} n_{10}+Z_{2} n_{20}\right)$ and $T_{\mathrm{c}}$ and $T_{\mathrm{h}}$, respectively. The corresponding solution of the initial value problem reads

$$
\begin{aligned}
f^{e} & =\frac{n_{\mathrm{c} 0}}{\sqrt{2 \pi} v_{T \mathrm{c}}} \exp \left(-I^{(\mathrm{c})} / v_{T \mathrm{c}}^{2}\right) \\
+ & \frac{n_{\mathrm{h} 0}}{\sqrt{2 \pi} v_{T \mathrm{~h}}} \exp \left(-I^{(\mathrm{h})} / v_{T \mathrm{~h}}^{2}\right), \\
f^{1} & =\frac{n_{10}}{\sqrt{2 \pi} v_{T 1}} \exp \left(-I^{(1)} / v_{T 1}^{2}\right), \\
f^{2} & =\frac{n_{20}}{\sqrt{2 \pi} v_{T 2}} \exp \left(-I^{(2)} / v_{T 2}^{2}\right),
\end{aligned}
$$

where $v_{T \alpha}^{2}=T_{\alpha} / m_{\alpha}$ and the invariants $I^{(\alpha)}$ are defined as follows:

$$
\begin{gathered}
\frac{I^{(\mathrm{c})}}{v_{T \mathrm{c}}^{2}}=\mathscr{E}+\frac{\left(1+\Omega^{2} t^{2}\right)}{2 v_{T \mathrm{c}}^{2}}(v-u)^{2}, \\
\frac{I^{\mathrm{h})}}{v_{T \mathrm{~h}}^{2}}=\mathscr{E} \frac{T_{\mathrm{c}}}{T_{\mathrm{h}}}+\frac{\left(1+\Omega^{2} t^{2}\right)}{2 v_{T h}^{2}}(v-u)^{2},
\end{gathered}
$$




$$
\begin{aligned}
\frac{I^{(1)}}{v_{T 1}^{2}}= & -\mathscr{E}\left(\frac{Z_{1} T_{\mathrm{c} 0}}{T_{10}}\right)+\frac{U^{2}}{2 v_{T 1}^{2}}\left(1+\frac{Z_{1} m_{e}}{m_{1}}\right) \\
& +\frac{\left(1+\Omega^{2} t^{2}\right)}{2 v_{T 1}^{2}}(v-u)^{2}, \\
\frac{I^{(2)}}{v_{T 2}^{2}}= & -\mathscr{E}\left(\frac{Z_{2} T_{\mathrm{c}}}{T_{2}}\right)+\frac{U^{2}}{2 v_{T 2}^{2}}\left(1+\frac{Z_{2} m_{e}}{m_{2}}\right) \\
& +\frac{\left(1+\Omega^{2} t^{2}\right)}{2 v_{T 2}^{2}}(v-u)^{2} .
\end{aligned}
$$

Here, $u=x t \Omega^{2} /\left(1+\Omega^{2} t^{2}\right)$ is the local plasma velocity; $U=x \Omega / \sqrt{1+\Omega^{2} t^{2}}$. The potential $\Phi$ is defined in the implicit form via the function $\mathscr{E}$,

$$
\mathscr{E}=\frac{e \Phi}{T_{\mathrm{c}}}\left(1+\Omega^{2} t^{2}\right)+\frac{U^{2}}{2 v_{T \mathrm{c}}^{2}},
$$

which obeys the transcendental equation,

$$
\begin{gathered}
1+\sigma-\rho_{\mathrm{h}}=\exp \left[\left(1+\frac{Z_{1} T_{\mathrm{c}}}{T_{1}}\right) \mathscr{E}-\frac{U^{2}}{2 v_{T 1}^{2}}\left(1+\frac{Z_{1} m_{e}}{m_{1}}\right)\right] \\
-\rho_{\mathrm{h}} \exp \left[\left(1-\frac{T_{\mathrm{c}}}{T_{\mathrm{h}}}\right) \mathscr{E}\right] \\
+\sigma \exp \left[\left(1+\frac{Z_{2} T_{\mathrm{c}}}{T_{2}}\right) \mathscr{E}-\frac{U^{2}}{2 v_{T 2}^{2}}\left(1+\frac{Z_{2} m_{e}}{m_{2}}\right)\right], \\
\rho_{\mathrm{h}}=\frac{n_{\mathrm{h} 0}}{Z_{1} n_{10}}, \quad \sigma=\frac{Z_{2} n_{20}}{Z_{1} n_{10}} .
\end{gathered}
$$

The solution to this equation allows one to define the dependences of the particle distribution functions on velocity, coordinate and time.

Equations (8)-(11) give exhaustive information on the kinetics of plasma foil expansion. However, rough integral characteristics might be more useful for practical applications. Two integral characteristics (such as partial ion density, $n_{q}(t, x)$, and ion energy spectra, $\left.d N_{q} / d \epsilon\right)$ can be calculated from the ion distribution functions:

$$
\begin{gathered}
n_{q}=\int_{-\infty}^{\infty} d v f^{q}, \\
\frac{d N_{q}}{d \epsilon}=\frac{1}{m_{q} v} \int_{-\infty}^{\infty} d x\left(f^{q}(t, x, v)+f^{q}(t, x,-v)\right) .
\end{gathered}
$$

When integrated over the energy, $\epsilon=m_{q} v^{2} / 2$, the value $d N_{q} / d \epsilon$ defines the total number of ions of the given species in a plasma slab per unit square.
The ion density is defined by the universal function $\mathcal{N}_{q}$ as follows:

$$
\begin{gathered}
n_{q}=\frac{n_{q 0}}{\sqrt{1+\Omega^{2} t^{2}}} \mathcal{N}_{q}(U), \\
\mathcal{N}_{q}(U)=\exp \left(\frac{\left.Z_{q} T_{\mathrm{c}} \mathscr{E}-\frac{U^{2}\left(1+Z_{q} m_{e} / m_{q}\right)}{T_{q}}\right) .}{2 v_{T q}^{2}}\right)
\end{gathered}
$$

The general form of $d N_{q} / d \epsilon$ given by Eqs. (8) and (12) is rather complicated, but its asymptotic behavior at $\Omega t \longrightarrow \infty$ is described by the simple expression

$$
\frac{d N_{q}}{d \epsilon} \approx \sqrt{\frac{2}{m_{q}} \epsilon} \frac{n_{q 0}}{\Omega} \mathcal{N}_{q}\left(\epsilon=\frac{m_{q} U^{2}}{2}\right) .
$$

Here, the functions $\mathcal{N}_{q}(U)$ are also given by Eq. (13), provided the ion energy $\epsilon$ is not very small, viz, $2 \epsilon / T_{q} \gg$ $(\Omega t)^{-2}$. The asymptotical spectrum of accelerated ions is similar to a Maxwellian spectrum, $\propto \exp \left(-v^{2}\right)$, rather than to the spectrum $\propto \exp (-v)$ corresponding to the theory of isothermal plasma expansion [43], which does take account of particle cooling during plasma expansion. The latter is an important effect, since the physics of plasma foil expansion differ from the physics of semi-infinite plasma expansion, where the isothermal regime is permitted because of the infinite source of plasma energy.

To illustrate the aforesaid, we present in Fig. 16 the dimensionless carbon and proton densities $n_{1} / n_{\mathrm{c} 0}(\mathrm{C})$ and $n_{2} / n_{\mathrm{c} 0}(\mathrm{H})$ as functions of the dimensionless coordinate $x^{2} / L_{0}^{2}$ for different times. This example corresponds to $\mathrm{CH}$ plasma expansion with $\mathrm{H}^{+1}$ and $\mathrm{C}^{+4}$ ions. We assume all ions have the same initial temperature $T_{1,2} / T_{\mathrm{c}}=0.1$ and that the hot to cold electron temperature ratio is $T_{\mathrm{h}} / T_{\mathrm{c}}=1000$. The concentration of hot electrons is $\rho_{\mathrm{h}}=5 \times 10^{-4}$. The left-hand panel shows the ion densities for $t=0.5\left(L_{0} / c_{s}\right)$; the right-hand panel, for $t=$ $2\left(L_{0} / c_{s}\right)$. The dashed lines present the benchmarks related to the densities of cold electrons, $n_{\mathrm{c}} / n_{\mathrm{c} 0}$ (short dashes), and hot electrons, $n_{\mathrm{h}} / n_{\mathrm{c} 0}$ (long dashes), respectively.

Figure 17 shows the evolution of the dimensionless ion energy spectra, $\left(m_{1} Z_{1} T_{\mathrm{c}} / 2\right)^{1 / 2}\left(\Omega / n_{10}\right)\left(d N_{q} / d \epsilon\right)$, for the same parameters as in Fig. 16. According to Fig. 17, the distribution $\left(d N_{q} / d \epsilon\right) \sqrt{\epsilon}$ definitely takes the form $\mathcal{N}_{q}(\epsilon)$ for $\Omega t \gg 1$. The left-hand panel corresponds to $\Omega t=0.5$ and the right-hand panel to $\Omega t=2$, respectively. Both energy spectra for light and heavy ions experience a cutoff on the high-energy side of the spectra. Note that the heavy ion component affects the energy spectrum of the light ions by means of a selfconsistent electric field, so that the proton spectrum has a maximum at the energy cutoff in the spectrum of car- 

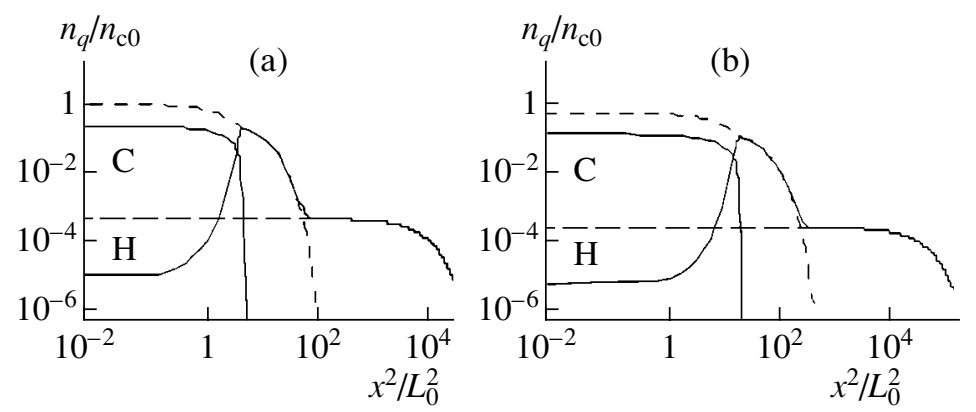

Fig. 16. Dimensionless densities of carbon ions (C) and protons (H) calculated by formulas (13) for (a) $t=0.5\left(L_{0} / c_{s}\right)$ and (b) $t=$ $2\left(L_{0} / c_{s}\right)$.
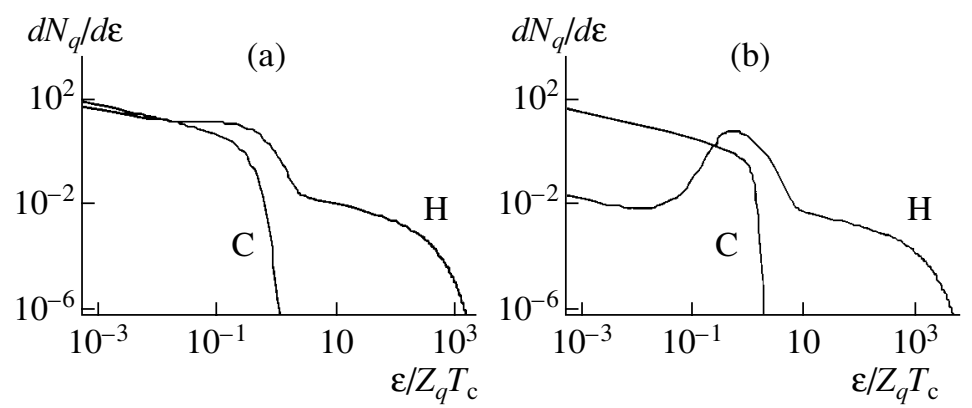

Fig. 17. Energy spectra $d N_{q} / d \epsilon$ of carbon ions (C) and protons (H) calculated by formulas (8) and (12) for (a) $t=0.5\left(L_{0} / c_{s}\right)$ and (b) $t=2\left(L_{0} / c_{s}\right)$.

bon ions. The proton energy cutoff settles at the level of the hot electron temperature, $\epsilon_{\max } \sim T_{\mathrm{h}}$.

Going beyond the model of quasineutral plasma expansion (which often cannot be used to interpret experiments on ion acceleration in expanding plasmas heated by ultra short laser pulses), we turn to the numeric Vlasov-Poisson model [49]. It uses the kinetic description of ions in a self-consistent electric field; this satisfies the Poisson equation, unlike the hydrodynamic model of [50]. Our Vlasov-Poisson model is well suited for modeling plasmas with many ion species.

As above, we include in this model two electron species: cold and hot. Arguing in favor of electron equilibration with the electrostatic potential on a time scale that is short compared to the plasma expansion time, most theoretical models assume that each electron species follows the Boltzmann distribution [36-38, 4042]. We also follow this assumption, although (as was shown before) the deviations from the equilibrium electron distributions may have some quantitative effect on the parameters of the accelerated ions.

As mentioned above, we now consider both electron components (cold and hot) to be in a quasi-equilibrium state following Boltzmann distribution with temperatures $T_{\mathrm{c}}$ and $T_{\mathrm{h}}$. Given the experimental data, one may refer to $T_{\mathrm{c}} \sim 1-2 \mathrm{keV}$ and $T_{\mathrm{h}} \sim 1-10 \mathrm{MeV}$ as the typical values. For short laser pulses of relativistic intensity, the hot electron temperature scales with a laser inten- sity quite close to the $\sqrt{I}$ dependence [51-53], so that the parameter $T_{\mathrm{h}}$ in our approach can serve as a connecting link to the laser, together with the number of hot electrons, $n_{\mathrm{h}}$, which depends on the laser pulse energy rather than its intensity. Considering $T_{\mathrm{h}}$ and $n_{\mathrm{h}}$ as the controlling laser parameters, we aim to characterize the ion acceleration that depends on them and on the parameters of a plasma such as its thickness, temperature, and ion composition.

We consider a symmetrical plasma slab $(-L<x<L)$ with an initially stepped ion density profile (at $x= \pm L$ ) that expands in the $\mathrm{x}$-direction into the vacuum. Plasma expansion in a self-consistent electric field, $-\partial_{x} \Phi$, is described by the Vlasov equations for ions (2); the electrons follow Boltzmann distributions

$$
n_{\mathrm{h}, \mathrm{c}}=n_{\mathrm{h} 0, \mathrm{c} 0} \exp \left(e \Phi / T_{\mathrm{h}, \mathrm{c}}\right) \text {, }
$$

where $n_{\mathrm{c} 0, \mathrm{~h} 0}$ are the electron densities in the unperturbed plasma, and, instead of Eq. (3), the electric potential $\Phi$ satisfies the Poisson equation

$$
\partial_{x x} \Phi
$$

$$
=4 \pi e\left[n_{\mathrm{h} 0} \exp \left(e \Phi / T_{\mathrm{h}}\right)+n_{\mathrm{c} 0} \exp \left(e \Phi / T_{\mathrm{c}}\right)-\sum_{\alpha} Z_{\alpha} n_{\alpha}\right] .
$$



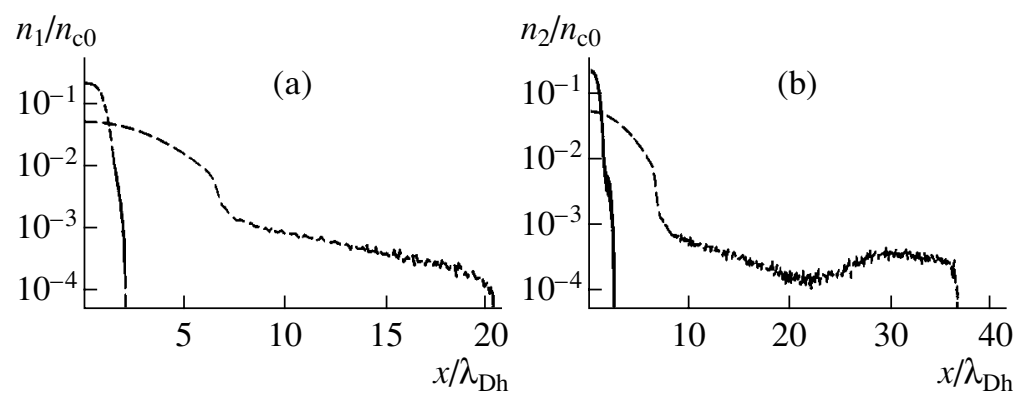

Fig. 18. Dimensionless densities of (a) carbon ions and (b) protons calculated by the Vlasov-Poisson model at $\omega_{p 2} t=1$ (solid lines) and $\omega_{p 2} t=10$ (dashed lines).
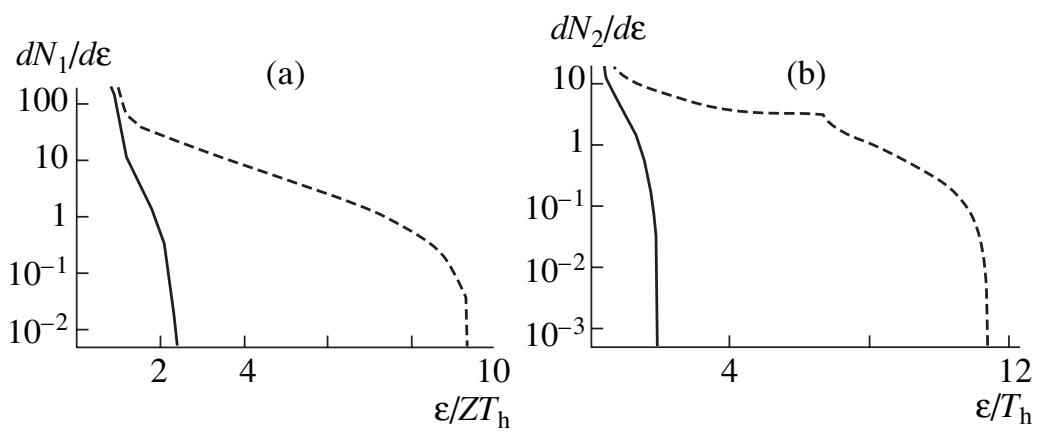

Fig. 19. Energy spectra $d N_{q} / d \epsilon$ of (a) carbon ions and (b) protons calculated by the Vlasov-Poisson model at $\omega_{p 2} t=1$ (solid lines) and $\omega_{p 2} t=10$ (dashed lines).

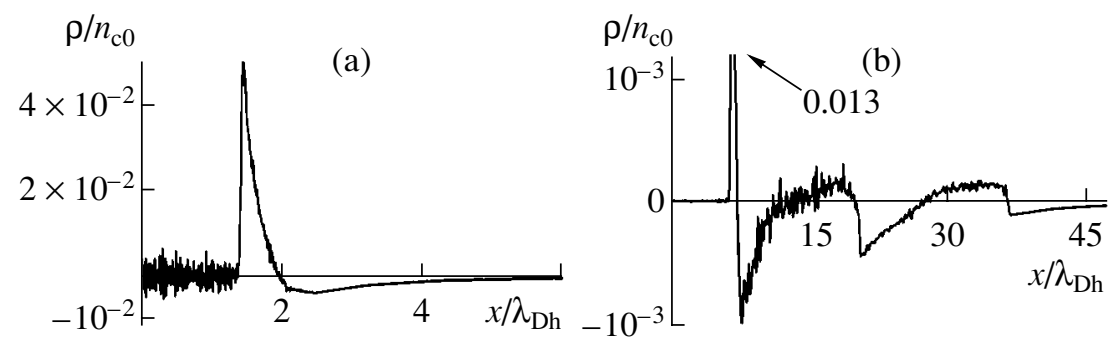

Fig. 20. The normalized space charge $\rho / n_{\mathrm{c} 0}=\left(Z_{1} n_{10}+Z_{2} n_{20}-n_{\mathrm{c} 0}-n_{\mathrm{h} 0}\right) / n_{\mathrm{c} 0}$ calculated by the Vlasov-Poisson model at (a) $\omega_{p 2} t=1$ and (b) $\omega_{p 2} t=10$, where 0.013 indicates the maximum of $\rho / n_{\mathrm{c} 0}$.

The results of the numerical solutions of Eqs. (2), (15), and (16) are shown in Figs. 18, 19, and 20 for a thin $\mathrm{CH}$ plasma slab of a half-thickness equal to the Debye length of the hot electrons $\left(L=\lambda_{\mathrm{Dh}}\right)$. All initial plasma parameters were the same as in above example of RGS theory, except for the hot electron density, which was $\rho_{\mathrm{h}}=2 \times 10^{-3}$. Figures 18 and 19 show, respectively, the densities and energy spectra of heavy $\left(\mathrm{C}^{4+}\right)$ and light $\left(\mathrm{H}^{+}\right)$ions. As should be, the protons that are accelerated by hot electrons form a halo in front of expanding plasma. At times $\omega_{p 2} t \gg 1$, where $\omega_{p 2}$ is the light ion plasma frequency, a band structure appears in the proton energy spectrum (Fig. 19) as in the analytical theory of quasineutral plasma (Fig. 17). At these times, the plasma is already close to a quasineutral state, although it is violated at the ion fronts of the fast protons, fast carbon ions, and bulk ions (Fig. 20). It is important to note that the maximum that precedes the cutoff in the proton energy spectrum (Fig. 19) is formed by protons that acquire additional acceleration at the heavy ion front, where the electric field has a maximum. This is entirely an effect of multi-species ion composition that can not appear in single-species plasma [50].

Unlike the theory of quasineutral plasma expansion, the high-energy part of the proton spectrum is more extended, exhibiting a proton tail with $\epsilon \gg T_{\mathrm{h}}$. We 
believe there are two reasons for this. First, the charge separation field, which is not included in the RGS model, increases the efficiency of ion acceleration. The charge-separation electric field peaks at the heavy ion front and effectively accelerates the light ions. Second, in contrast, the electron cooling that is allowed for in the analytic theory is not included in the numeric Vlasov-Poisson model and may result in the overestimation of the maximum proton energy. Such cooling is expected to be important if the laser pulse-duration is shorter than the typical acceleration time, which is several $\omega_{p 2}^{-1}$.

As a final note in this section, we mention that hot electrons control the ion acceleration if $n_{\mathrm{h} 0} T_{\mathrm{h}}>n_{\mathrm{c} 0} T_{\mathrm{c}}$. In this case the accelerating electrostatic field can be estimated from Eq. (16) as follows:

$$
E \simeq \sqrt{8 \pi n_{\mathrm{h} 0} T_{\mathrm{h}}} .
$$

For hot electron temperature $\sim 1 \mathrm{MeV}$, this corresponds to Eq. (1) if the hot electron density is about the $10^{20} \mathrm{~cm}^{-3}$ that is typical for our experiments. As times goes by, the accelerating electric field (17) forms peaks at the ion fronts where the field amplitudes decrease over time as $t^{-1}$, similar to the results in [50].

\section{PARTICLE ACCELERATION IN PIC SIMULATIONS}

The models presented above suggest that the generation of high-energy electrons is crucial to ion acceleration. To self-consistently describe electron generation, 2D PIC simulations were performed by using the code in [54]. In this section, we present the results of such simulations for a 140-fs (FWHM) linearly polarized laser pulse with a wavelength $\lambda=1 \mu \mathrm{m}$, normally incident onto an underdense plasma slab having an exponential density profile with a spatial scale length $L=$ $5 \mu \mathrm{m}$ [52]. Behind this was a thin dense plasma slab with a density forty times higher than critical and thickness $d=0.25 \mu \mathrm{m}$. This thickness was ten times greater than the skin depth, so the plasma slab was not transparent to the laser pulse. We used such a preplasma to model the blow-off plasma created by the laser prepulse, which interacts with the foil before the main pulse reaches the target. A laser pulse with vacuum electric field amplitude $E_{y 0}$ propagates in the $x$ direction of an $(x, y)$ simulation plane and has a $3-\mu \mathrm{m}$ focal size. The normalized amplitude of the laser vector potential, $a=e E_{\mathrm{y} 0} / m c \omega_{0}$, was in the range $0.5 \leq a \leq 13.8$, corresponding to laser intensities $I \lambda^{2}$ of $3.3 \times 10^{17}$ to $2.5 \times$ $10^{20} \mathrm{~W} / \mathrm{cm}^{2} \mu \mathrm{m}^{2}$. The total simulation box was $50 \times$ $10 \mu \mathrm{m}$, and the dense plasma target was at $x=10 \mu \mathrm{m}$. A long vacuum region was situated behind the target to reduce boundary effects. We performed simulations at different preplasma scale lengths $(0<L / \lambda<10)$ and plasma target thicknesses $(0.1<d / \lambda<8)$ for a laser intensity of $a=2$. A hydrogen-deuterium plasma was chosen with $20 \% \mathrm{H}$ and $80 \% \mathrm{D}$.

Our choice of a relatively thin dense plasma slab was motivated mainly by the TW scale experiments with dielectric targets [10] to model front side ion generation. According to [16], the temperature of the bulk electrons in the solid dense plasma is around $1 \mathrm{keV}$ for such an experiment. Initially, only a thin skin layer less than $0.1 \mu \mathrm{m}$ in width is heated to this temperature. During heating, the size of the hot dense plasma increases due to the energy transport of the bulk electrons inside the target. However, the heat transport velocity, $\sim f_{V_{T e}}$, where $v_{T e}$ is the electron thermal velocity and $f$ is the heat flux inhibition factor, is rather low; with a subpicosecond laser pulse, a hot dense plasma cannot expand to greater than a micron in size. For example, at a pulse duration of $400 \mathrm{fs}$ [10] and the commonly used value of $f \sim 0.1$, one may estimate $d \simeq 1 \mu \mathrm{m}$. For the laser intensities considered here, the main laser energy is converted to hot electrons, which may penetrate at least to their Debye length. For instance, for a hot electron density of about $n_{\mathrm{cr}}$ and a temperature $\sim 1 \mathrm{MeV}$, the Debye length is $\sim 0.3 \lambda$. We follow Scenario 1 for the hot electron penetration of a highly resistive material, where the right plasma boundary is representative of the hot plasma-cold solid interface. This is likely to be relevant to the experiments in [10] with insulators. Measurements of the hot electron penetration depth [16] for the same parameters typical of the experiments in [10] have shown a rather short penetration depth of $<3 \mu \mathrm{m}$. In order to model deeper energy deposition (due, for example, to a prepulse), we changed $d$ in our simulations by as much as $8 \lambda$.

Thus, ion acceleration is governed entirely by the high-energy electrons generated in the preplasma. At the same time, electron motion is very complicated because of the combined effect of the pump, the reflected and self-generated fields, and the plasma inhomogeneity. In Fig. 21, we show a 2D-distribution of the electromagnetic energy and quasistatic fields (the fields averaged over the laser period): the magnetic field $\left(B_{z}\right)$ and the longitudinal electrostatic field $\left(E_{x}\right)$ for a subrelativistic laser intensity, $a=1$. The reflection of laser light produces a standing wave, which is seen in Fig. 21 to be a modulation of the laser intensity on the scale of $\lambda / 2$. While the laser beam forms a well-pronounced channel in the corona, the self-focusing was insignificant for the given parameters. Surface currents and the electrons expelled backwards cause a magnetic field generated by the so-called "fountain effect," with a maximum of up to $40 \%$ of the laser field (Fig. 21b).

The good correlation between the laser intensity and the modulations of the longitudinal electric field in the preplasma can be seen clearly in Fig. 21c. We have concluded that the latter is due to the ponderomotive effect at each maximum of the laser intensity. However, in an arbitrary $y$-slice, the electrostatic field exhibits chaotic behavior (Fig. 21d) that cannot be seen in Fig. 21c 

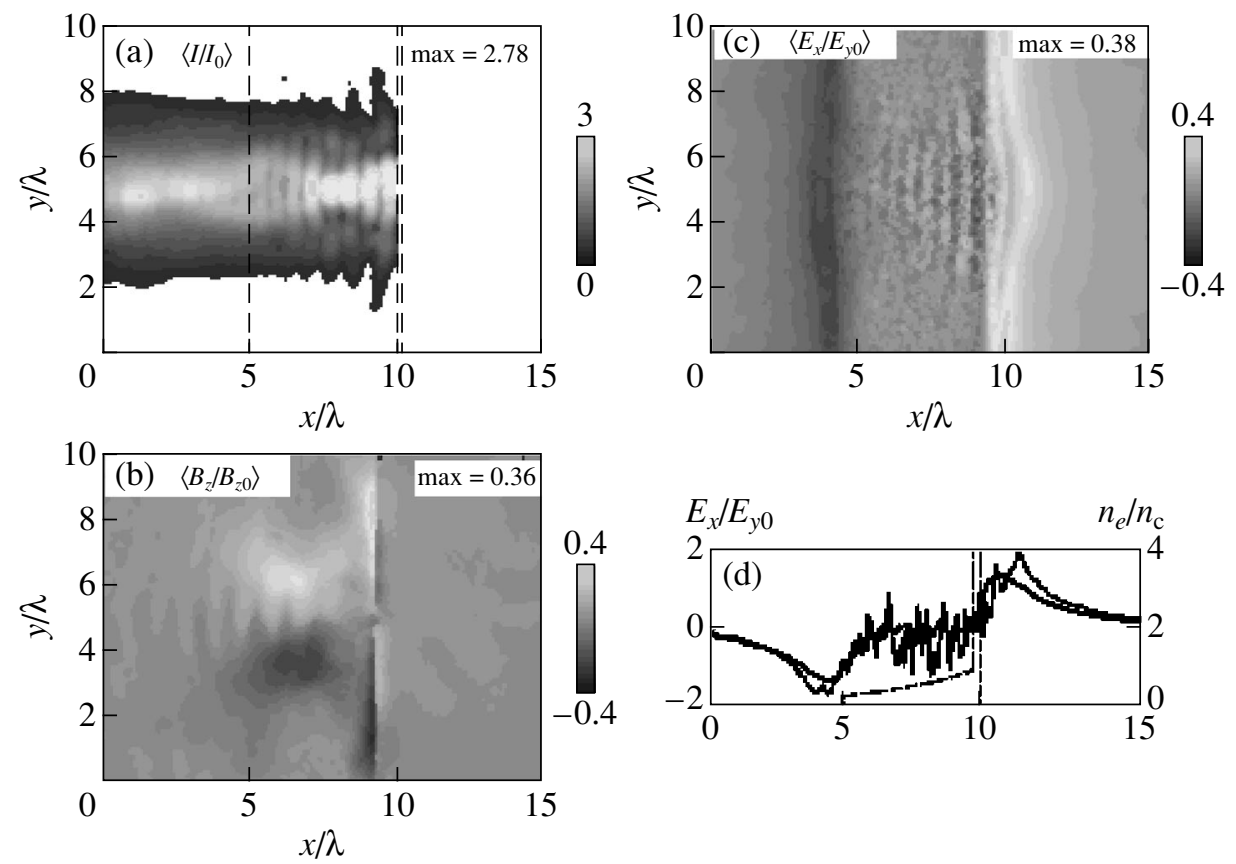

Fig. 21. The electromagnetic energy at $t=106 \mathrm{fs}$ (a), the quasistatic magnetic field at $t=185 \mathrm{fs}$ (b), the longitudinal electrostatic field at $t=185 \mathrm{fs}(\mathrm{c})$, and the corresponding slice plot at $y=5 \mu \mathrm{m}(\mathrm{d})$ for dimensionless laser field $a=1$. Dashed lines indicate the initial plasma boundaries. All plots are time averaged over the laser period.
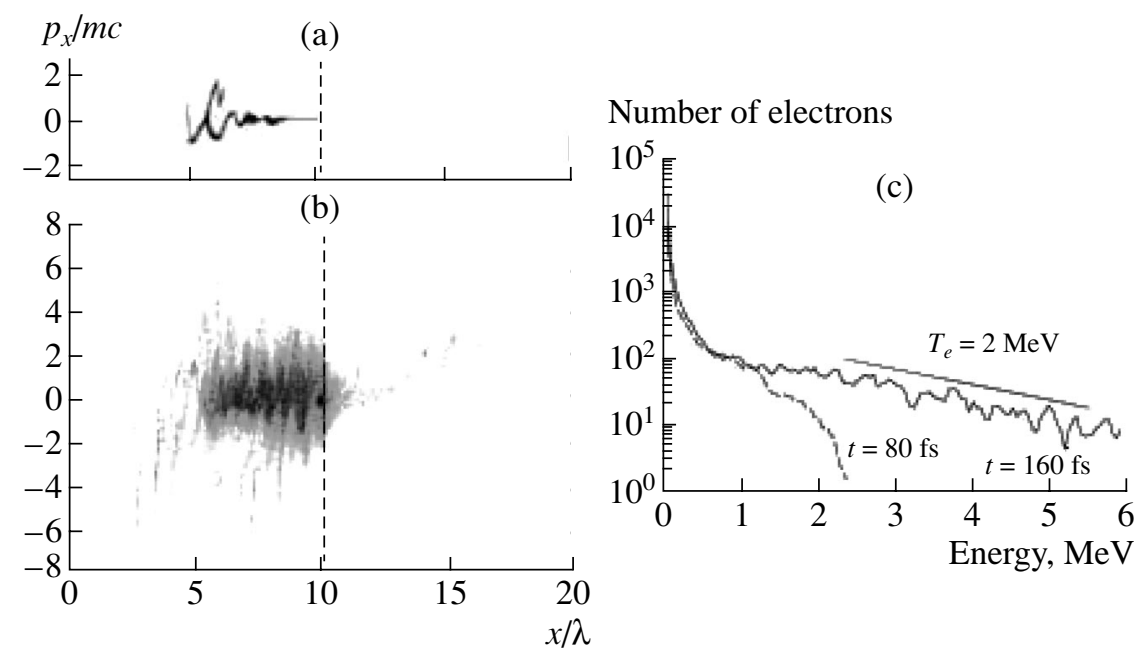

Fig. 22. The electron phase space plots $\left(x, p_{x}\right)$ during the laser pulse, at (a) $t=40 \mathrm{fs}$ and (b) $t=80$ fs. The energy distribution of forward accelerated electrons (c) during the laser pulse, at $t=80 \mathrm{fs}$, and after it, $t=160 \mathrm{fs}$. Laser intensity corresponds to $a=1$.

because of the rather small scale of the electrostatic fluctuations across the laser beam. On the other hand, averaged over the laser focal size (in order to filter out noise), this electrostatic field shows a well defined periodic structure in the $x$ direction with a spatial scale of $\lambda / 2$. The electron density has similarly strong modulations. Hence, the acceleration of electrons occurs in preplasma with a very complicated electromagnetic field structure and density. Propagating through the tar- get, these electrons produce a strong charge separation field which reaches $40 \%$ of the laser field behind the target and smoothly decreases with distance, as shown in Fig. 21d.

The PIC model gives details of the electron energy distribution, as is shown in Fig. 22 for $a=1$. The phase space plots (Figs. 22a, 22b) show space modulations on the scale of $\lambda / 2$ due to the formation of a standing electromagnetic wave; this plays an important role in elec- 


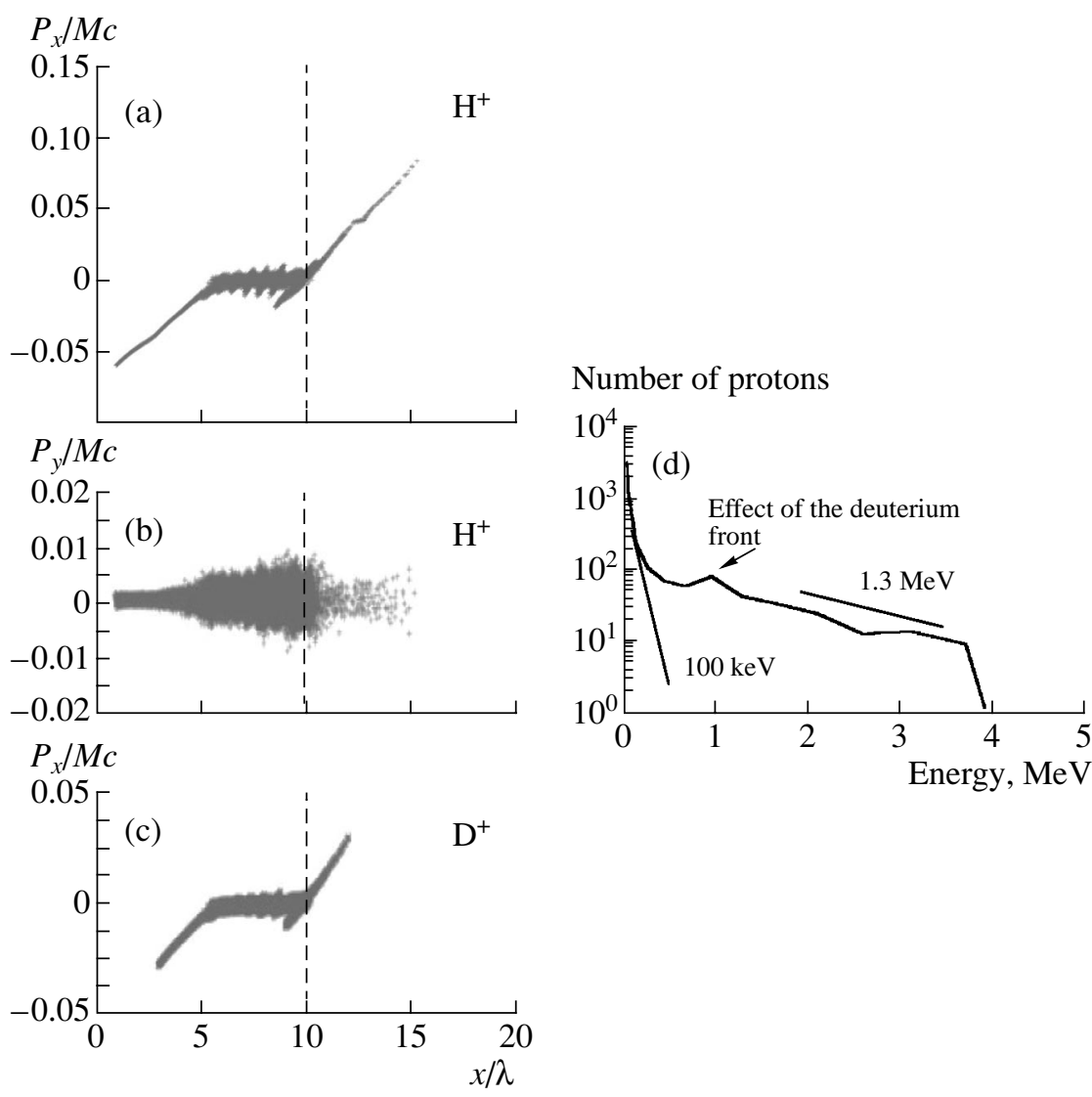

Fig. 23. The simulation results for $a=1$ : the ion phase space plots $\left(x, p_{x}\right)$ (a) and (c) for $\mathrm{H}^{+}$and $\mathrm{D}^{+}$ions, respectively, and $\left(x, p_{y}\right)$ (b) for $\mathrm{H}^{+}$ions at $t=330 \mathrm{fs}$. The energy distribution of forward accelerated protons is shown in the panel (d) at $t=330 \mathrm{fs}$. Dashed vertical line shows the position of thin overdense plasma slab; $M$ is the ion mass for protons and deuterons, respectively.

tron heating. On this run, $60 \%$ of the laser energy was reflected; i.e., the reflectivity coefficient related to the field amplitude was $r \sim 0.8$. For $a \sim 1$, the amplitude of the longitudinal momentum oscillations due to the formation of the standing electromagnetic wave can be estimated at $p_{x} / m c \sim(1+r)^{2} a^{2} \sim 3$, (cf. Fig. 22b). Over several laser cycles, the electron motion becomes stochastic. Thus, we conclude that for our simulations with a rather extended preplasma, stochastic electron heating is the dominant process for electron energy gain. Stochastic heating originates from the standing wave formation due to the reflection of laser light. This was proved by the simple numeric model presented in [52], where it was shown that stochastic electron heating occurs when $r>0.2$.

The PIC simulations suggest that the preplasma, which can be attributed to the finite intensity contrast ratio of the laser pulse, makes the laser capable of producing ions in the $\mathrm{MeV}$ range at $I>5 \times 10^{17} \mathrm{~W} / \mathrm{cm}^{2}$. In Figs. 23a and 23b, the longitudinal and transverse proton momenta are shown versus distance $x$. Comparison between 23a and 23b demonstrates that the high-energy protons are expelled as two beams, one in the forward direction and one in the backward direction, and the energy of the forward accelerated ions is higher. The energy of the deuterons is four to five times lower than that of the protons (Fig. 23c). Deuterons are two times heavier than protons and have less mobility; following behind the protons, they experience a significantly lower accelerating electric field and therefore gain less energy.

Figure 23d shows the ion energy spectrum for $a=1$. It exhibits a low energy (100-200 keV) ion bulk and a hot ion tail (1.0-1.5 MeV) that transitions smoothly to a flat distribution with a sharp energy cutoff (4 MeV). Such a flat distribution with an energy cutoff is typical for an electrostatic mechanism of ion acceleration [2-4, $6,55-57]$. It corresponds to the maximum electric potential produced by the hot electrons. One more piece of evidence for the electrostatic nature of ion acceleration is provided by the peak in the proton distribution at an energy of $\sim 1 \mathrm{MeV}$; this corresponds to the deuteron front and defines in turn the self-consistent accelerating field.

From our study of ion trajectories, we conclude that the maximum energy gain is obtained when the protons are accelerated from the back of an overdense plasma, in accordance with the spatial distribution of the elec- 


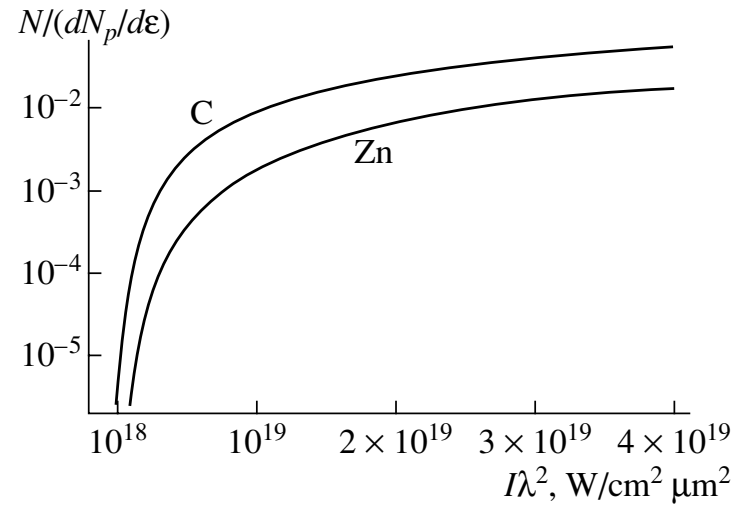

Fig. 24. The yields $N /\left(d N_{p} / d \epsilon\right)$ of ${ }^{11} \mathrm{~B}(p, n){ }^{11} \mathrm{C}$ and ${ }^{63} \mathrm{Cu}(p, n){ }^{63} \mathrm{Zn}$ reactions versus laser intensity.

trostatic field (Fig. 21d). Indeed, the electric field is maximum behind the dense plasma slab so that the rear side protons respond first and the others that follow behind them are accelerated by a screened potential. However, this result needs to be reconciled with the contradictory experimental observations of ion acceleration from either the front $[2,6]$ or the rear surfaces $[3$, 4]. In experiments with a high intensity contrast ratio $[2,10]$, the rear surface of a solid dense plasma is in fact the front surface of a foil, because the thickness of the highly ionized region is negligible compared to the foil thickness. This is likely not the case for the experiments in which a rear-surface origin was found [3,4], where a very intense prepulse preceded the main laser pulse.

\section{COMPARISON WITH EXPERIMENTS}

The PIC simulations agree with the features of highenergy ion emission observed in our experiments. By studying the dependence of the maximum ion energy $\epsilon_{\max }$ on the laser intensity $I\left(3.3 \times 10^{17} \leq I \leq 2.5 \times\right.$ $10^{20} \mathrm{~W} / \mathrm{cm}^{2}$ ), we found $\epsilon_{\max } \propto I$ and $\epsilon_{\max } \propto \sqrt{I}$ dependences [52] for two regimes with $a \leq 1$ and $a>1$, respectively. The physical picture behind these $I$ and $\sqrt{I}$ dependences is quite simple and is based on the generation of hot electrons in the preformed plasma. The electrons interacting with an electromagnetic wave of $\omega_{0}>c k_{0}$ acquire an energy $m c^{2}(\gamma-1)$, where $\gamma$ is the relativistic factor. This gives the scalings $m c^{2} a^{2}$ and $m c^{2} a$ for subrelativistic and ultrarelativistic laser intensities, respectively. During stochastic heating (Section 7), a quasi-thermal electron energy distribution forms with an effective temperature $T_{\mathrm{h}}$, which scales as $m c^{2}(\gamma-1) \propto$ $\min \left\{a^{2}, a\right\} m c^{2}$. Hence, the electrostatic potential of the charge-separation field and the ion energy both have the same dependences on the laser intensity. The latter agrees qualitatively with Fig. 3, with the dependence quite close to the $\sqrt{I}$ dependence for high intensities

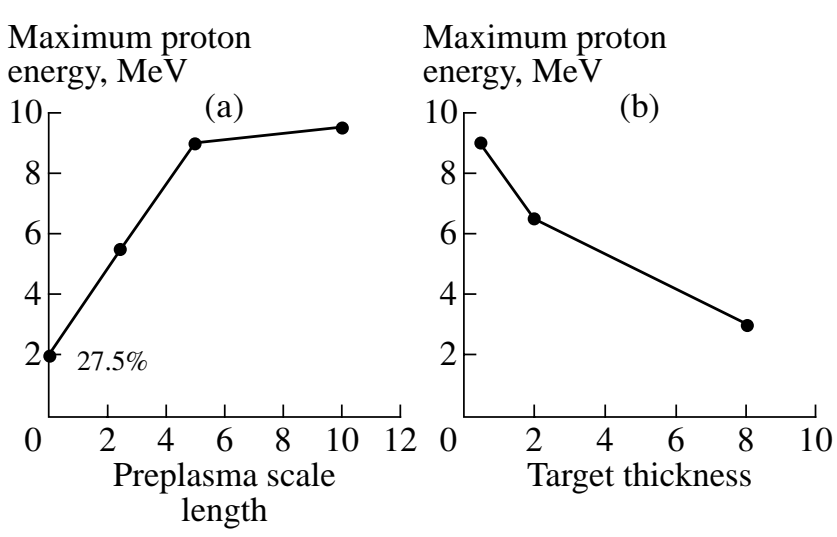

Fig. 25. Maximum proton energy versus (a) the preplasma scale length and (b) the overdense plasma thickness (in units of $\lambda$ ) for $a=2$.

suggested in [57]. Given our simulation and experimental results and the experimental results for higher intensities in $[3,57]$, one finds that the maximum proton energy for the relativistic laser intensity is well approximated as [58]

$$
\epsilon_{\max }[\mathrm{MeV}] \approx 3.6 \lambda \sqrt{I / 10^{18}}
$$

where the laser intensity is in $10^{18} \mathrm{~W} / \mathrm{cm}^{2}$ units and the laser wavelength is in $\mu \mathrm{m}$.

The plateau-like energy distribution of fast ions (Figs. 2, 5) agrees with both theory and our simulations (Sections 6, 7). Along with this, Fig. 2 demonstrates a Maxwellian-like distribution of the bulk protons, similar to Eq. (14), indicating an adiabatic regime of plasma expansion [46, 47, 59] rather than an isothermal one [43]. Note that proton spectra with band structures, as shown in Fig. 34, are also tolerated by theoretical models (Fig. 17, 19). Similar band structures of the proton energy spectra were also observed in the experiments in $[61,62]$. That the production of protons is more efficient than that of heavy ions (Figs. 17, 19, 23c) is confirmed by the $(d, n)$ activation experiment. Our simulations quantify the inhibition of deuteron energy when they are accelerated along with protons [10], as described in Section 4. The experimental data on the activation of a boron sample with deuterons at $I=6 \times$ $10^{18} \mathrm{~W} / \mathrm{cm}^{2}$ suggest that their energy was $\simeq 2 \mathrm{MeV}$, while the proton energy was as high as $8 \mathrm{MeV}$. This is close to the simulation result that yields a deuteron energy four times lower than the proton energy.

Because of the plateau-like energy distribution of fast protons, the reaction yield can be easily estimated by using the so-called overlap integral [62]

$$
N \approx \frac{1}{M} \frac{d N_{p}}{d \epsilon} \int_{0}^{\epsilon_{\max }} d \epsilon\left(\epsilon_{\max }-\epsilon\right) \frac{\sigma(\epsilon)}{S(\epsilon)},
$$

where one may use Eq. (18) for the proton energy cutoff. This expression quantifies the number of reactions 
versus the laser field intensity in a nuclear target with atoms of mass $M$, where laser accelerated protons react with target nuclei in a layer of thickness equal to their stopping length $S[\mathrm{MeV} \mathrm{cm} / \mathrm{g}]$ with the efficiency defined by their cross section $\sigma$. In Eq. (19) the value $d N_{p} / d \epsilon$ is the number of high-energy (multi-MeV) protons per $1 \mathrm{MeV}$ range. Figure 24 shows the nuclear yield (19), normalized to the number of fast protons in the $1 \mathrm{MeV}$ range as a function of laser intensity. The thresholds at $10^{18}$ and $2 \times 10^{18} \mathrm{~W} / \mathrm{cm}^{2}$ for ${ }^{11} \mathrm{~B}(p, n){ }^{11} \mathrm{C}$ and ${ }^{63} \mathrm{Cu}(p, n)^{63} \mathrm{Zn}$ reactions are clearly seen in this figure. Because the measured number of protons with energy $>(3-4) \mathrm{MeV}$ was $\sim 10^{7}-10^{8}$ per $1 \mathrm{MeV}$ range, the production of $\sim 10^{5}$ atoms of ${ }^{11} \mathrm{C}$ and ${ }^{63} \mathrm{Zn}$ follows from Eq. (19); this accords with the results of our activation experiments (Section 4).

In Section 2, the existence of an optimum in the laser prepulse intensity was demonstrated for the maximum ion energy gain. The maximum proton energy shown by the experimental points in Fig. 1b increases with the prepulse intensity for a contrast ratio $>10^{3}$ and then decreases. This is in qualitative agreement with the simulation results demonstrated in Fig. 25. It is likely that at the rather low intensity $I \sim 10^{18} \mathrm{~W} / \mathrm{cm}^{2}$, the scale length of the preformed underdense plasma grows with the intensity of the prepulse without a significant increase in the volume of the overdense plasma if the prepulse intensity remains sufficiently low. Accordingly, the maximum ion energy should increase and then saturate (Fig. 25a). However, at higher prepulse intensities, one might expect enhanced solid-density plasma production before the main laser pulse reaches the target. As a result, the efficiency of the ion acceleration decreases (Fig. 25b) due to the reduction in the average density of the hot electrons that participate in ion acceleration. We predict the saturation of ion energy when the solid plasma thickness exceeds the laser pulse length (if hot electron losses in the target are small).

The interplay between these two effects should result in a dependence similar to the experimental one shown in Fig. 1b, with the maximum at some moderate contrast ratio. An ion energy decrease and saturation with the target thickness was recently observed in an experiment [13] with a high energy laser.

\section{NEAR FUTURE REGIMES OF PARTICLE ACCELERATION}

As powerful short-pulse laser installations tend toward the multi-PW level and tabletop lasers continue to reach record levels of peak intensity, we look forward to the next physical regimes of ion acceleration. The first regime, which is expected for quite highpower lasers of ultra-relativistic intensities, might be called the nuclear regime of laser-plasma interactions [63], because of the generation of such elementary particles as mesons and baryons that should occur as nuclei collide in such energetic plasmas. The second regime
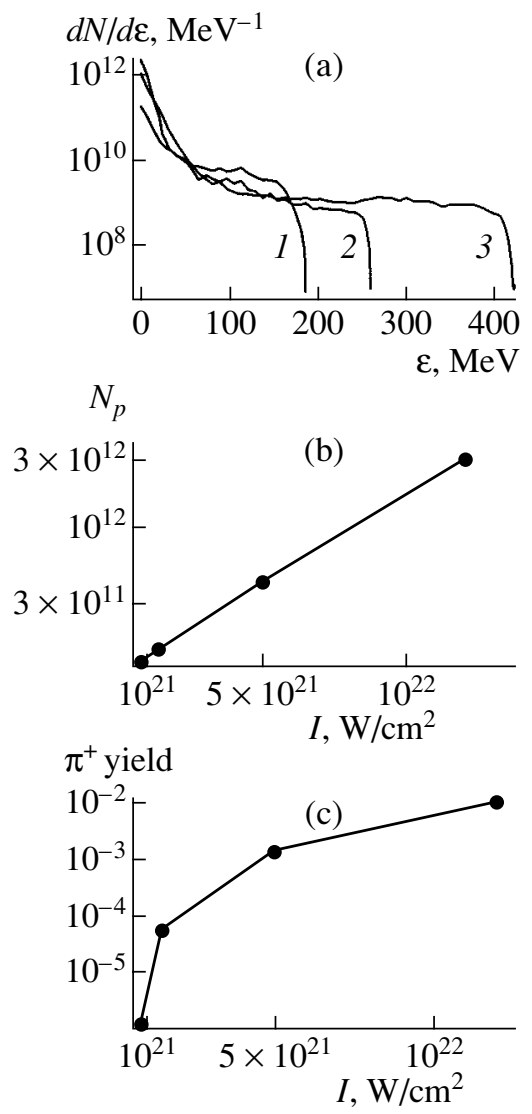

Fig. 26. (a) Energy distribution of the forward accelerated protons for laser intensities (1) $8 \times 10^{20}$, (2) $1.4 \times 10^{21}$, and (3) $5 \times 10^{21} \mathrm{~W} / \mathrm{cm}^{2}$; (b) the number of forward accelerated protons with energy exceeding $140 \mathrm{MeV}$ versus laser intensity; and (c) pion yield per a proton from the proton collisions with the $\mathrm{C}$ nuclei as a function of laser intensity.

deals with compact and ultrashort pulse-duration (several optical cycles) lasers, which are focused in a single-wavelength spot size to operate at relativistic intensities. The great advantage of these lasers is that they work at high repetition rates. One prototype of such a device is the CPA laser at CUOS [53]. This indirectly diode-pumped Ti:sapphire laser works at a $1-\mathrm{kHz}$ repetition rate and has the advantage of being stable (1\% variation), compact (it fits on a single $8^{\prime} \times 10^{\prime}$ table), highly-intense (with an energy several times greater than $\left.10^{18} \mathrm{~W} / \mathrm{cm}^{2}\right)$ at a rather low level of energy $(\leq 3 \mathrm{~mJ})$ and a relatively low cost. Further improvement of this apparatus will provide a strongly relativistic intensity, $I=10^{19} \mathrm{~W} / \mathrm{cm}^{2}$.

We have demonstrated for the first time the nuclear regime of laser-plasma interactions by pion production from accelerated protons [62]. Using 2D PIC simulations, we modeled the interaction of a short 300-fs linearly polarized pulse with a solid $5-\mu \mathrm{m}$-thick foil representing a uniform plasma layer with a density forty times higher than its critical value. Laser radiation with a wavelength of $1 \mu \mathrm{m}$ was incident normally on the foil 

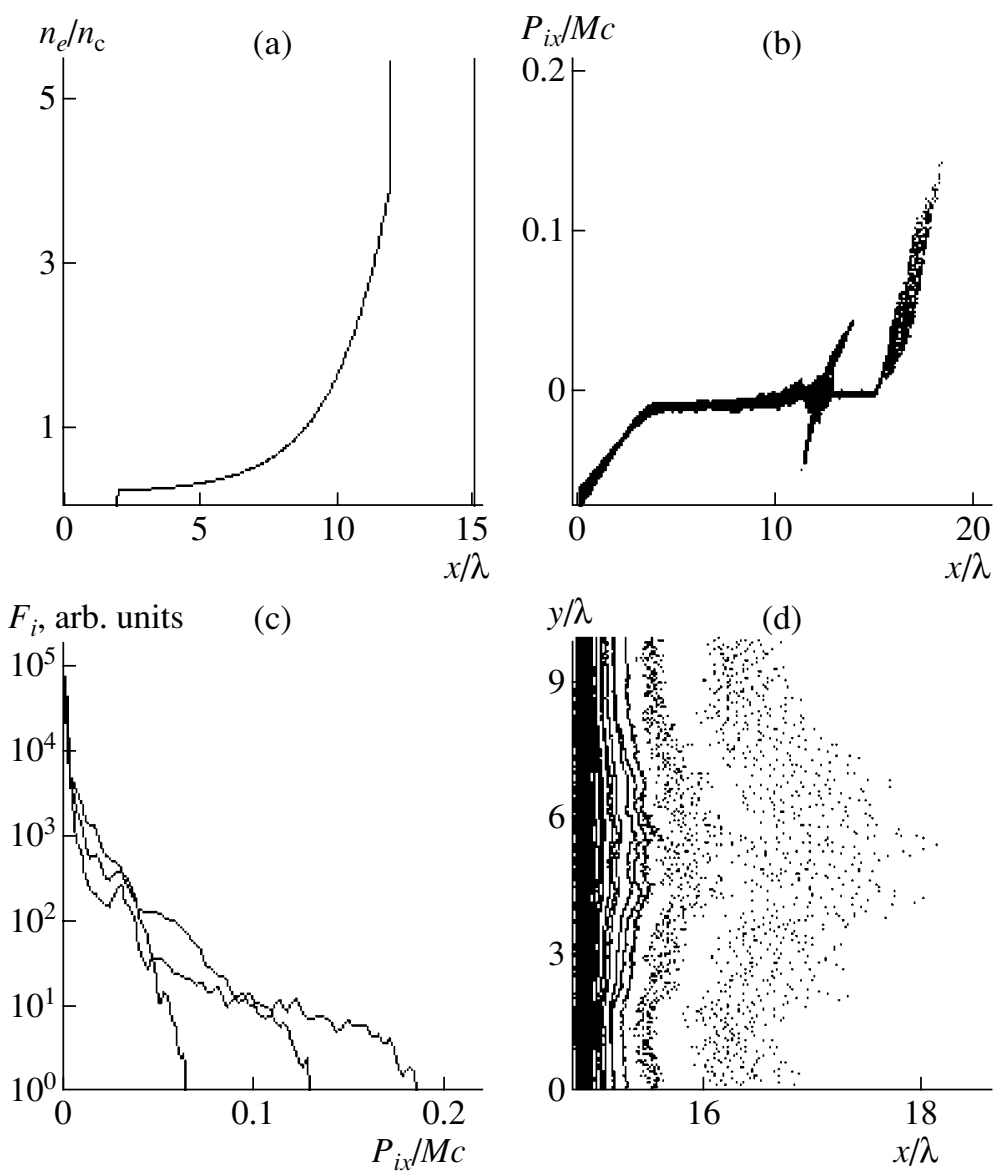

Fig. 27. (a) Initial electron density profile; (b) the ion phase space plot $\left(x, p_{i x}\right)$ at $\omega_{0} t / 2 \pi=90$; (c) the ion momentum distribution functions at $\omega_{0} t / 2 \pi=40,70$, and 90; and (d) spatial distribution of accelerated ions behind the foil at $\omega_{0} t / 2 \pi=70$.

surface. The focused spot size was $3 \mu \mathrm{m}$, while laser intensity varied in the range of $2 \times 10^{20}$ to $5 \times$ $10^{21} \mathrm{~W} / \mathrm{cm}^{2}$. To model a preplasma from a prepulse, a $30-\mu \mathrm{m}$-thick layer of rarefied plasma was placed in front of the foil. These simulations revealed plateaulike proton distributions with energy cutoffs similar to those presented in Section 7, but at much higher energies. Evidently, once the cutoff in the proton spectrum exceeds $140 \mathrm{MeV}$, protons can produce $\pi^{+}$in the material behind the foil.

In Fig. 26, the energy spectra of the forward accelerated protons, the number of accelerated protons $N_{p}$ with energy higher than $140 \mathrm{MeV}$, and the pion yield from a carbon target placed behind the foil are shown.

The number of high-energy protons that may produce $\pi^{+}$increases in almost direct proportion to the laser intensity. Our calculations can be used to predict the laser intensity threshold for pion production. This threshold is clearly seen in Fig. 26 at $I_{\text {th }} \sim 10^{21} \mathrm{~W} / \mathrm{cm}^{2}$. At $I=5 \times 10^{21} \mathrm{~W} / \mathrm{cm}^{2}$, the pion production yield is $10^{-3}$. For $4 \times 10^{11}$ accelerated protons, this provides a value of $4 \times 10^{8}$ particles per shot for the total $\pi^{+}$yield, which is five orders of magnitude higher than the photopion yield obtained under similar conditions in [64]. Pions can be identified most simply from the $\pi \mu$ decay using the standard method, because the lifetime of a $\pi^{+}$is short, $\tau_{\pi}=26 \mathrm{~ns}$. At a pulse repetition rate of $1 \mathrm{kHz}$, laser muon production will offer advantages over conventional accelerator methods and provide a muon flux of $10^{14}-10^{15} \mathrm{~s}^{-1}$.

In current laser techniques, the pion production threshold intensity of $10^{21} \mathrm{~W} / \mathrm{cm}^{2}$ can certainly be reached using low-energy lasers, provided that the pulse duration equals several femtoseconds. However, there is a fundamental limitation that requires a considerably long pulse duration: during the proton acceleration time $\tau_{i} \sim \sqrt{m_{p} / e^{2} n_{\mathrm{h}}}$, the laser must provide a permanent source of hot electrons. Otherwise, they cool down and are not able to accelerate ions efficiently (Section 6). Estimating the density of the hot electrons as the critical density, one obtains $\tau_{i} \sim 100$ fs for $1-\mu \mathrm{m}$ laser. On the other hand, the smallest attainable focal spot size for high-power lasers is equal to several microns. Hence, it follows that, to produce pions, the laser output should be as high as several tens of joules. 
In the above example of pion production in proton collisions with nuclei, the pion distribution is nearly isotropic. At the same time, for hydrogen-containing nuclear targets where pions may be produced in $p-p$ collisions, one can expect the generation of pion and neutrino beams, provided that the proton energy far exceeds $1 \mathrm{GeV}$, i.e., at $I>10^{23} \mathrm{~W} / \mathrm{cm}^{2}$. The lifetime of pions with energies of up to several $\mathrm{GeV}$ is considerably longer than $\tau_{\pi}=26 \mathrm{~ns}$. This opens the way to their subsequent acceleration by the laser plasma method of particle acceleration in an underdense plasma [65] in order to produce ultrahigh-energy pions, which are as yet present only in cosmic rays. However, in contrast to the latter, the laser generation of ultrahigh energy pions is predictable. Note that one may consider laser-triggered muon production as a potential source for the socalled Muon Spin Rotation/Relaxation/Resonance ( $\mu$ SR) technique [66]. The $\mu$ SR technique is now used to tackle on an atomic scale fundamental problems in condensed matter physics and chemistry that cannot be investigated by other means.

Finally, we turn to ion acceleration by ultra-short laser pulses, only one order of magnitude longer than the laser period, focused into very small spots with sizes comparable to the laser wavelength. A prototype of such a table top laser is now in operation at CUOS within the limits of a pulse duration of only a few cycles and a single-wavelength spot size. Our 2D PIC simulations using the UMKA code developed by the Institute of Computational Technologies [67, 68] were performed for a linearly polarized laser pulse propagating normally to a plasma target (hydrogen) in the $x$ direction of an $(x, y)$ simulation plane. This target models a solid dense plasma slab of thickness $3 \lambda$, with a rare plasma in front of it to simulate the blow-off plasma created by the laser prepulse that interacts with the foil before the main pulse reaches the target. The dense plasma slab has an electron density forty times higher than critical. The total simulation box is $40 \lambda \times 10 \lambda$. The preplasma is at $x>2 \lambda$ with an electron density that grows from $0.25 n_{\text {cr }}$ to $4 n_{\text {cr. }}$ The laser pulse propagates in the $x$ direction from left to right with a vacuum electric field dimensional amplitude $a=7$. It has the small focal size $2 \lambda$ and length $12 \lambda$. The position of the laser beam axis corresponds to $y=5 \lambda$. There is a $2 \lambda$ vacuum layer before the plasma target and a significantly longer vacuum region behind it to reduce boundary effects. Our PIC model used 50 particles per cell.

The short laser pulse effectively generates electrons in the preplasma. The plasma density profile, which is shown in Fig. 27a, is not extraordinary and has a characteristic size of $10 \lambda$. However, it calls for about $50 \%$ of the laser energy to be transformed into electrons with an energy $\geq 3 \mathrm{MeV}$; these penetrate through the target and accelerate ions up to $18 \mathrm{MeV}$. This is demonstrated in Figs. $27 b$ and 27c, in which the ion phase space plot and distribution function are shown. The ions escape from the rear side of the foil as a well collimated jet
(Fig. 27d). The total numbers of fast electrons and protons with an energy $\geq 3 \mathrm{MeV}$ are $\sim 10^{11}$ and $\sim 10^{10}$, respectively. Although lasers with a pulse duration $\leq 100$ fs are not optimal for high-energy ion generation [63], this provides hope that a laser with an energy of $100-200 \mathrm{~mJ}$ can be used to initiate most conventional nuclear reactions through the practical use of fast protons. For instance, the simulation performed allows us to predict that a radioactive ${ }^{11} \mathrm{C}$ source can gain around $1 \mathrm{Ci}$ by using Ti:sapphire laser with an energy of $\sim 100 \mathrm{~mJ}$ and a $100-\mathrm{Hz}$ repetition rate.

\section{SUMMARY}

We have presented here the results from our experimental advances in the generation of high-energy ions in the interaction of a high-intensity short pulse laser with thin film targets. The characteristics of highenergy ions were measured and their correlation with laser intensity, wavelength, intensity contrast ratio, and the material and shape of the target were found. Highenergy deuterons and protons were used to perform experiments on nuclear transformations ${ }^{10} \mathrm{~B}(d, n){ }^{11} \mathrm{C}$, ${ }^{11} \mathrm{~B}(p, n){ }^{11} \mathrm{C}$, and ${ }^{63} \mathrm{Cu}(p, n){ }^{63} \mathrm{Zn}$. Using shaped targets, we observed proton beam focusing and found that the target conductivity has a considerable impact on the quality of the generated proton beams. The experiments performed shed light on the means intended for the optimization of laser-based ion sources.

Either because of imperfect laser compression or amplified spontaneous emission, background light accompanies a short intense pulse and arrives at the focus first. For the current laser intensities $10^{19}$ $10^{21} \mathrm{~W} / \mathrm{cm}^{2}$ and typical contrast ratios $10^{5}-10^{6}$ the corresponding prepulse intensity is as high as $10^{14}$ $10^{16} \mathrm{~W} / \mathrm{cm}^{2}$, which is more than enough for plasma creation. Typically, such a prepulse lasts from several hundreds of picoseconds to a few nanoseconds. Hence, the peak of a high intensity laser pulse arrives after the onset of plasma creation and expansion, and deposits its energy in an underdense long-density-scalelength plasma, rather than directly at the solid density. Numerous mechanisms of hot electron generation take place in such an underdense plasma; these are crucial for high-energy ion generation. We believe that preplasma formation should be taken into account in experimental and theoretical studies of ion acceleration. The manipulation of the preplasma in terms of its size and density profile, as well as arranging for optimum laser-plasma matching (including self-focusing in a preplasma), are important factors in creating a high-energy ion source with desirable properties.

The physical models of ion acceleration by fast electrons presented here provide an easy-to-use tool for estimating ion characteristics by means of their electron parameters. The corresponding correlation measurements might be an interesting subject for experiments on laser triggered ions. The 2D PIC simulations 
performed qualitatively agree with the experiments. Using PIC simulations, we obtained scalings for ion energy with laser intensities $I$ and $\sqrt{I}$ for the sub- and relativistic regimes, and described the dependences of ion generation on both the preformed and overdense plasma sizes. The observed increase of the maximum ion energy with greater preplasma thickness and its saturation demonstrates that an ideal laser pulse with a very high contrast ratio is not optimal for effective high-energy ion generation. The latter appears to be a result of the suppression of hot electron generation in a short-density scalelength underdense plasma. Finally, our PIC simulations predict the threshold of pion production for a Petawatt-class laser and the threshold of $\mathrm{MeV}$ ion generation for a table-top laser with a pulse duration of several cycles and a single-wavelength spot size.

One may expect that the development of laserdriven ion acceleration will result in several important applications. If protons were laser accelerated to 100$150 \mathrm{MeV}$ energies, they could be useful for proton therapy, which is now limited to cyclotrons and the associated large magnets required to transport the proton beams to the patient. Protons are superior to other forms of ionizing radiation for cancer treatment due to their lower straggling and their ability to precisely deposit their energy in tissue. Protons and deuterons with energies of several $\mathrm{MeV}$ could be useful for isotope production. The production of short-lived isotopes in micro-samples using a high repetition rate laser might be promising for medicine, biology, and educational purposes. Laser-accelerated proton beams could be used as a tool for radiography and imaging in material science and for fundamental nuclear research on the picosecond time scale. A laser-based meson factory is also seen as one future application of laser- accelerated proton beams with energy above $150 \mathrm{MeV}$.

\section{ACKNOWLEDGMENTS}

The authors would like to thank T. Lin and M. Rever for their help with the CR-39 processing. This work was partly supported by the U.S. National Science Foundation (a FOCUS Grant), the Russian Foundation for Basic Research (project nos. 03-02-16428 and 02-01-00185), and INTAS (grant nos. 01-0233 and 01-0572).

\section{REFERENCES}

1. E. L. Clark, K. Krushelnick, J. R. Davies, et al., Phys. Rev. Lett. 84, 670 (2000).

2. A. Maksimchuk, S. Gu, K. Flippo, et al., Phys. Rev. Lett. 84, 4108 (2000).

3. S. P. Hatchett, C. G. Brown, T. E. Cowan, et al., Phys. Plasmas 7, 2076 (2000).

4. R. A. Snavely, M. H. Key, S. P. Hatchett, et al., Phys. Rev. Lett. 85, 2945 (2000).
5. S. J. Gitomer, R. D. Jones, F. Begay, et al., Phys. Fluids 29, 2679 (1986).

6. K. Krushelnick, E. L. Clark, M. Zepf, et al., Phys. Plasmas 7, 2055 (2000).

7. Yu Wei, V. Bychenkov, Y. Sentoku, et al., Phys. Rev. Lett. 85, 570 (2000).

8. Y. Sentoku, T. V. Liseikina, T. Zh. Esirkepov, et al., Phys. Rev. E 62, 7271 (2000).

9. S. V. Bulanov, N. M. Naumova, T. Zh. Esirkepov, et al., Pis'ma Zh. Éksp. Teor. Fiz. 71, 593 (2000) [JETP Lett. 71, 407 (2000)].

10. K. Nemoto, A. Maksimchuk, S. Banerjee, et al., Appl. Phys. Lett. 78, 595 (2001).

11. N. Izumi, Y. Sentoku, H. Habara, et al., Phys. Rev. E 65, 036413 (2002).

12. M. Zepf, E. L. Clark, F. N. Beg, et al., Phys. Rev. Lett. 90, 064801 (2003).

13. A. J. Mackinnon, M. Borghesi, S. Hatchett, et al., Phys. Rev. Lett. 86, 1769 (2001).

14. J. Badziak, E. Woryna, P. Parys, et al., Phys. Rev. Lett. 87, 215001 (2001).

15. A. J. Mackinnon, Y. Sentoku, P. K. Patel, et al., Phys. Rev. Lett. 88, 215006 (2002).

16. Z. Jiang, J. C. Kieffer, J. P. Matte, et al., Phys. Plasmas 2, 1702 (1995).

17. L. Gremillet, F. Amiranoff, S. D. Baton, et al., Phys. Rev. Lett. 83, 5015 (1999).

18. A. R. Bell, J. R. Davies, S. Guerin, and H. Ruhl, Plasma Phys. Controlled Fusion 39, 653 (1997).

19. J. R. Davies, A. R. Bell, M. G. Haines, and S. M. Guerin, Phys. Rev. E 56, 7193 (1997).

20. J. R. Davies, A. R. Bell, and M. Tatarakis, Phys. Rev. E 59, 6032 (1999).

21. F. Pisani, A. Bernardinello, D. Batani, et al., Phys. Rev. E 62, 5927 (2000).

22. M. Hegelich, S. Karsch, G. Pretzler, et al., Phys. Rev. Lett. 89, 085002 (2002).

23. M. V. Ammosov, N. B. Delone, and V. P. Kraŭnov, Zh. Éksp. Teor. Fiz. 91, 2008 (1986) [Sov. Phys. JETP 64, 1191 (1986)].

24. Experimental Nuclear Reaction Data File http://www.nndc.bnl.gov/nndc/exfor.

25. G. S. Sarkisov, V. Yu. Bychenkov, and V. T. Tikhonchuk, Pis'ma Zh. Éksp. Teor. Fiz. 69, 20 (1999) [JETP Lett. 69, 20 (1999)].

26. Kh. Rul', S. V. Bulanov, T. E. Cowan, et al., Fiz. Plazmy 27, 387 (2001) [Plasma Phys. Rep. 27, 363 (2001)].

27. S. V. Bulanov and V. S. Khoroshkov, Fiz. Plazmy 28, 493 (2002) [Plasma Phys. Rep. 28, 453 (2002)].

28. S. C. Wilks, A. B. Langdon, T. E. Cowan, et al., Phys. Plasmas 8, 542 (2001).

29. M. Borghesi, A. Schiavi, D. H. Campbell, et al., Plasma Phys. Controlled Fusion 43, A267 (2001).

30. M. Borghesi, D. H. Campbell, A. Schiavi, et al., Phys. Plasmas 9, 2214 (2002).

31. M. Borghesi, S. Bulanov, D. H. Campbell, et al., Phys. Rev. Lett. 88, 135002 (2002).

32. A. J. Mackinnon, P. K. Patel, D. W. Price, et al., Rev. Sci. Instrum. 74, 1917 (2003). 
33. A. J. Mackinnon, P. K. Patel, D. W. Price, et al., Appl. Phys. Lett. 82, 3188 (2003).

34. S. C. Wilks, W. L. Kruer, M. Tabak, and A. B. Langdon, Phys. Rev. Lett. 69, 1383 (1992).

35. E. J. Valeo and I. B. Bernstein, Phys. Fluids 19, 1348 (1976).

36. B. Bezzerides, D. W. Forslund, and E. L. Lindman, Phys. Fluids 21, 2179 (1978).

37. L. M. Wickens, J. E. Allen, and P. T. Rumsby, Phys. Rev. Lett. 41, 243 (1978).

38. L. M. Wicken and J. E. Allen, J. Plasma Phys. 22, 167 (1979).

39. J. Denavit, Phys. Fluids 22, 1384 (1979).

40. A. Gurevich, D. Anderson, and H. Wilhelmsson, Phys. Rev. Lett. 42, 769 (1979).

41. M. A. True, J. R. Albritton, and E. A. Williams, Phys. Fluids 24, 1885 (1981).

42. L. M. Wickens and J. E. Allen, Phys. Fluids 24, 1894 (1981).

43. A. V. Gurevich, L. V. Pariı̆skaya, and L. P. Pitaevskiŭ, Zh. Éksp. Teor. Fiz. 49, 647 (1965) [Sov. Phys. JETP 22, 449 (1965)].

44. C. Sack and H. Schamel, Phys. Rep. 156, 311 (1987).

45. D. S. Dorozhkina and V. E. Semenov, Phys. Rev. Lett. 81, 2691 (1998).

46. V. F. Kovalev, V. Yu. Bychenkov, and V. T. Tikhonchuk, Pis'ma Zh. Éksp. Teor. Fiz. 74, 12 (2001) [JETP Lett. 74, 10 (2001)].

47. V. F. Kovalev, V. Yu. Bychenkov, and V. T. Tikhonchuk, Zh. Éksp. Teor. Fiz. 122, 264 (2002) [JETP 95, 226 (2002)].

48. V. F. Kovalev, V. V. Pustovalov, and D V. Shirkov, J. Math. Phys. 39, 1170 (1998).

49. V. Yu. Bychenkov, D. Batani, G. I. Dudnikova, et al., in Book of Abstracts of the 11th International Conference on Laser Optics, St. Petersburg, 2003, p. 29.

50. P. Mora, Phys. Rev. Lett. 90, 185002 (2003).
51. A. G. Zhidkov, A. Sasaki, I. Fukumoto, et al., Phys. Plasmas 8, 3718 (2001).

52. Y. Sentoku, V. Yu. Bychenkov, K. Flippo, et al., Appl. Phys. B 74, 207 (2002).

53. G. I. Dudnikova, V. Yu. Bychenkov, A. Maksimchuk, et al., Phys. Rev. E 67, 026416 (2003).

54. Y. Sentoku, H. Ruhl, K. Mima, et al., Phys. Plasmas 6, 2855 (1999).

55. G. S. Sarkisov, V. Yu. Bychenkov, V. N. Novikov, et al., Phys. Rev. E 59, 7042 (1999).

56. A. Pukhov, Z. M. Sheng, and J. Meyer-ter-Vehn, Phys. Plasmas 6, 2847 (1999).

57. E. L. Clark, K. Krushelnick, M. Zepf, et al., Phys. Rev. Lett. 85, 1654 (2000).

58. V. Yu. Bychenkov, V. Rozmus, A. Maksimchuk, et al., Fiz. Plazmy 27, 1076 (2001) [Plasma Phys. Rep. 27, 1017 (2001)].

59. V. F. Kovalev and V. Yu. Bychenkov, Phys. Rev. Lett. 90, 185004 (2003).

60. R. Decoste and B. H. Ripin, Phys. Rev. Lett. 40, 34 (1978).

61. M. Allen, Y. Sentoku, P. Audebert, et al., Phys. Plasmas 10, 3283 (2003).

62. V. Yu. Bychenkov, Y. Sentoku, S. V. Bulanov, et al., Pis'ma Zh. Éksp. Teor. Fiz. 74, 664 (2001) [JETP Lett. 74, 586 (2001)].

63. D. Umstadter, Phys. Plasmas 8, 1774 (2001).

64. S. Karsch, D. Habs, T. Schatz, et al., Laser Part. Beams 17, 565 (1999).

65. T. Tajima and J. M. Dawson, Phys. Rev. Lett. 43, 267 (1979).

66. http://musr.triumf.ca.

67. S. V. Bulanov, V. A. Vshivkov, G. I. Dudnikova, et al., Fiz. Plazmy 23, 284 (1997) [Plasma Phys. Rep. 23, 259 (1997)].

68. S. V. Bulanov, V. A. Vshivkov, G. I. Dudnikova, et al., Fiz. Plazmy 25, 764 (1999) [Plasma Phys. Rep. 25, 701 (1999)]. 